This item was submitted to Loughborough's Research Repository by the author.

Items in Figshare are protected by copyright, with all rights reserved, unless otherwise indicated.

\title{
The van Hove distribution function for Brownian hard spheres: Dynamical test particle theory and computer simulations for bulk dynamics.
}

\section{PLEASE CITE THE PUBLISHED VERSION}

http://dx.doi.org/10.1063/1.3511719

\section{PUBLISHER}

(C) the Authors. Published by AIP

VERSION

VoR (Version of Record)

\section{PUBLISHER STATEMENT}

This work is made available according to the conditions of the Creative Commons Attribution-NonCommercialNoDerivatives 4.0 International (CC BY-NC-ND 4.0) licence. Full details of this licence are available at: https://creativecommons.org/licenses/by-nc-nd/4.0/

\section{LICENCE}

CC BY-NC-ND 4.0

\section{REPOSITORY RECORD}

Hopkins, Paul, Andrea Fortini, Andrew J. Archer, and Matthias Schmidt. 2019. "The Van Hove Distribution Function for Brownian Hard Spheres: Dynamical Test Particle Theory and Computer Simulations for Bulk Dynamics.". figshare. https://hdl.handle.net/2134/26049. 


\section{The van Hove distribution function for Brownian hard spheres: Dynamical test particle theory and computer simulations for bulk dynamics}

Paul Hopkins, , Andrea Fortini, , Andrew J. Archer, and , and Matthias Schmidt

Citation: The Journal of Chemical Physics 133, 224505 (2010); doi: 10.1063/1.3511719

View online: $h$ ttp://dx.doi.org/10.1063/1.3511719

View Table of Contents: http://aip.scitation.org/toc/jcp/133/22

Published by the American Institute of Physics

\section{Articles you may be interested in}

Communication: Dynamical density functional theory for dense suspensions of colloidal hard spheres

The Journal of Chemical Physics 143, 181105 (2015); 10.1063/1.4935967

Dynamic pair correlations and superadiabatic forces in a dense Brownian liquid

The Journal of Chemical Physics 145, 064506 (2016); 10.1063/1.4960031

Equation of State for Nonattracting Rigid Spheres

The Journal of Chemical Physics 51, 635 (2003); 10.1063/1.1672048

Brownian dynamics with hydrodynamic interactions

The Journal of Chemical Physics 69, 1352 (2008); 10.1063/1.436761

Dynamic density functional theory of fluids

The Journal of Chemical Physics 110, 8032 (1999); 10.1063/1.478705

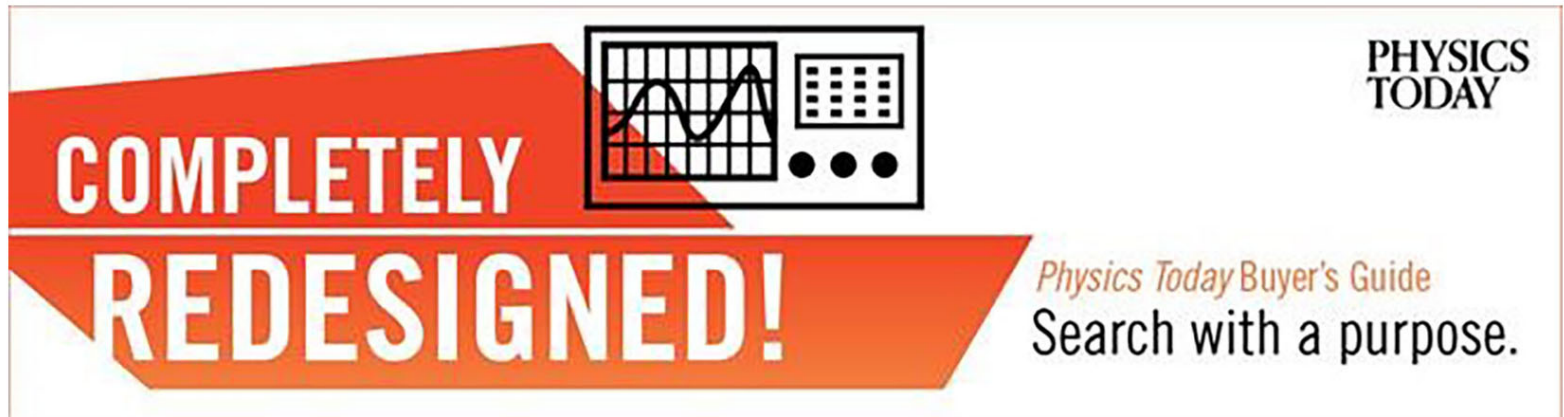




\title{
The van Hove distribution function for Brownian hard spheres: Dynamical test particle theory and computer simulations for bulk dynamics
}

\author{
Paul Hopkins, ${ }^{1}$ Andrea Fortini, ${ }^{2}$ Andrew J. Archer, ${ }^{3, a)}$ and Matthias Schmidt ${ }^{1,2}$ \\ ${ }^{1}$ H.H. Wills Physics Laboratory, University of Bristol, Tyndall Avenue, Bristol BS8 1TL, United Kingdom \\ ${ }^{2}$ Theoretische Physik II, Physikalisches Institut Universität Bayreuth, D-95440 Bayreuth, Germany \\ ${ }^{3}$ Department of Mathematical Sciences, Loughborough University, Loughborough LE11 3TU, United Kingdom
}

(Received 5 August 2010; accepted 17 October 2010; published online 13 December 2010)

\begin{abstract}
We describe a test particle approach based on dynamical density functional theory (DDFT) for studying the correlated time evolution of the particles that constitute a fluid. Our theory provides a means of calculating the van Hove distribution function by treating its self and distinct parts as the two components of a binary fluid mixture, with the "self" component having only one particle, the "distinct" component consisting of all the other particles, and using DDFT to calculate the time evolution of the density profiles for the two components. We apply this approach to a bulk fluid of Brownian hard spheres and compare to results for the van Hove function and the intermediate scattering function from Brownian dynamics computer simulations. We find good agreement at low and intermediate densities using the very simple Ramakrishnan-Yussouff [Phys. Rev. B 19, 2775 (1979)] approximation for the excess free energy functional. Since the DDFT is based on the equilibrium Helmholtz free energy functional, we can probe a free energy landscape that underlies the dynamics. Within the mean-field approximation we find that as the particle density increases, this landscape develops a minimum, while an exact treatment of a model confined situation shows that for an ergodic fluid this landscape should be monotonic. We discuss possible implications for slow, glassy, and arrested dynamics at high densities. (C) 2010 American Institute of Physics. [doi:10.1063/1.3511719]
\end{abstract}

\section{INTRODUCTION}

The structure of condensed matter is commonly probed by $\mathrm{x}$-ray or neutron scattering techniques that yield quantities such as $S(k)$, the static structure factor, and its dynamical counterpart $F(k, t)$, the intermediate scattering function. ${ }^{1}$ However, in recent years, with the advent of modern confocal microscopes, which are able to characterize the structure of colloidal suspensions in real space, there is an equally great emphasis on determining the radial distribution function $g(r)$ and its dynamical counterpart $G(r, t)$, the van Hove distribution function. ${ }^{1-3}$ The van Hove distribution function $G(r, t)$ is a real-space dynamical correlation function for characterizing the spatial and temporal distributions of pairs of particles in a fluid. It gives the probability of finding a particle at position $\mathbf{r}$ at time $t$, where $|\mathbf{r}|=r$, given that one of the particles was located at the origin at time $t=0$. The intermediate scattering function $F(k, t)$ is simply obtained from $G(r, t)$ via spatial (three-dimensional) Fourier transform. Pair correlation functions are important because of the significant amount of information that they contain. Transport coefficients can be calculated via Kubo formulae-for example, the diffusion coefficient $D$ can be obtained from $G(r, t)$-and thermodynamic quantities such as the internal energy and the pressure can be related to spatial integrals ${ }^{1}$ involving $g(r)$. Whether or not a liquid is near to freezing can often be discerned by inspecting the height of the principal peak in $S(k)$ : it was first noticed by Hansen and Verlet ${ }^{4}$ that many simple liquids freeze when the principal peak in $S(k)$ at $k=k_{m}$, takes the value

a) Electronic mail: A.J.Archer@lboro.ac.uk.
$S\left(k_{m}\right) \simeq 2.85$. Whether a system is a glass (i.e., an amorphous solid) rather than a fluid may be determined from the long time limit value of $F(k, t)$ because in a fluid the $t \rightarrow \infty$ limit of $F(k, t)$ is zero, whereas for a glass this limit takes nonzero values. This brief (and incomplete) survey is intended to demonstrate that both dynamical and static pair correlation functions are fundamental for characterizing and understanding liquids.

In the history of liquid state physics, fluids of hard spheres have proved to be an important model system for developing new techniques and theories. The hard sphere model is composed of particles interacting via the pair potential

$$
v_{h s}(r)= \begin{cases}\infty & r<\sigma \\ 0 & \text { otherwise }\end{cases}
$$

where $r$ is the distance between the centers of the particles and $\sigma$ is the hard sphere diameter. Hard spheres play an important role in describing real systems, because attractive interactions such as those present in the Lennard-Jones potential can often be treated as a perturbation to the hard sphere system. ${ }^{1}$ Hence a theory that can successfully describe the properties of the hard sphere fluid forms a good candidate to work for more realistic systems. The hard sphere model has further grown in importance in recent decades due to the fact that Eq. (1) provides a good model for the effective interaction potential between colloidal particles in suspension, in the case when the charges on the colloids are small or well screened-see e.g. Ref. 5 for an example of such a system. As the density of a hard sphere fluid is increased, the system freezes to a 
crystalline state, and the hard sphere model has played an important role in developing our understanding of this phase transition. In contrast, although the glass transition has attracted much interest in recent years, it is still not completely understood. An introduction to the vast literature on this subject can be found in Refs. 1 and 6, and references therein. A number of universal processes have been discovered, including dynamical heterogeneity, ${ }^{7}$ stretched exponential decay of correlation functions, ${ }^{8}$ and two-stage relaxation times. ${ }^{9}$ In order to understand the processes involved in structural arrest, a number of different theoretical approaches have been used. In particular, mode-coupling theory (MCT) has been successful in describing the bulk glass transition for hard sphere colloids, ${ }^{9}$ and has been applied, e.g., to suspension rheology. ${ }^{10,11}$ Nevertheless, alternatives to MCT have been developed. ${ }^{12-16}$ What is clear from the many studies of arrested systems is that key signatures of the slow dynamics are manifest in dynamical pair correlation functions.

In our previous Rapid Communication, ${ }^{17}$ a theory to calculate the van Hove function was proposed. For a bulk fluid of particles interacting via Gaussian pair potentials, comparison with Brownian dynamics (BD) computer simulation results showed that the theory is very reliable for determining $G(r, t)$ for this particular model system. The theory is formulated for inhomogeneous systems and hence was also applied to investigate the dynamics of hard spheres confined between two parallel hard walls. ${ }^{17}$ This approach has since been applied to investigate dynamics in liquid crystalline systems. ${ }^{18}$ In the current paper we explore the theory further, and apply it to study a bulk fluid of Brownian hard spheres. We present results for the self and distinct parts of the van Hove distribution function, $G_{s}(r, t)$ and $G_{d}(r, t)$, respectively, and by Fourier transforming, for the intermediate scattering function $F(k, t)$. We also display results for the scaled intermediate scattering function $\phi(k, t) \equiv F_{s}(k, t) / F_{s}(k, t=0)$ evaluated at the wave number $k \sigma=2 \pi$. This function is often the central object of focus of MCT. ${ }^{6}$ The two-stage relaxation of $\phi(k, t)$, that MCT predicts close to the glass transition, is also present in our theory. We also determine $G(r, t)$ and $F(k, t)$ using BD computer simulations and compare these results with those from the theory.

Our starting point is a dynamical generalization of Percus' test-particle approach ${ }^{19}$ for determining the radial distribution function $g(r)$. Percus showed that for a fluid of classical particles interacting via the pair interaction potential $v(r)$, that if one sets the external potential acting on the fluid $u(\mathbf{r})=v(r)$, then the one body density distribution $\rho(r)$ of the fluid around the fixed "test" particle is equal to the radial distribution function, multiplied by the bulk fluid density $\rho$; i.e., Percus showed that $\rho(r)=\rho g(r)$. When using equilibrium density functional theory (DFT) (Refs. 1 and 20) to study a fluid, the test-particle method provides a useful route to obtain $g(r)$ because $u(\mathbf{r})$ [and hence $v(r)$ ] enters the framework explicitly. We also describe an alternative "zero-dimensionality" approach for calculating $g(r)$. This forms a stepping stone in the development of the dynamical theory.

We apply a dynamical extension of Percus' idea, together with dynamical density functional theory (DDFT) (Refs. 2123 ) in order to calculate the van Hove function $G(r, t)$ in general inhomogeneous situations. We implement the method using the very simple Ramakrishnan-Youssouff (RY) approximation for the Helmholtz-free energy functional. ${ }^{24}$ We find that the results from the theory agree well with those from BD computer simulations when the fluid density $\rho \sigma^{3} \lesssim 0.6$. At higher densities the free energy underlying the dynamics develops a minimum, corresponding to the appearance of a free energy barrier that must be traversed for a particle to escape from the cage formed by the neighboring particles.

In addition, we compare our results for $G(r, t)$ to those obtained by assuming that $G_{s}(r, t)$ takes a simple Gaussian form for all times $t$, together with the Vineyard approximation, ${ }^{1,25}$ which sets $G_{d}(r, t)$ to be a simple convolution of $G_{s}(r, t)$ and the radial distribution function $g(r)$ as described in detail below. We find that in contrast to the received wisdom, ${ }^{26}$ the simple Vineyard approximation is actually a fairly good approximation for the van Hove function for Brownian hard sphere fluids at low and intermediate densities.

We also compare to an equilibrium DFT based approach with which we are able to calculate a series of density profiles that agree well with those from the DDFT. This is done by performing a constrained minimization of the free energy through the judicious choice of a suitable external potential to confine the test particle. This approach is easier to implement than the full DDFT and allows for the free energy landscape underlying the dynamics to be mapped out and examined in detail. However, this approach does not give any of the time information that the full DDFT gives-i.e., it yields the van Hove function with the time labels "removed." One of the advantages of this approach is that for a particular (parabolic) choice of external potentials, we are able to calculate exactly the fluid density profiles, which are precisely those predicted by Vineyard's theory. ${ }^{1,25}$ We discuss the significance of this result below, after we have laid out the general structure of the theory and shown the results.

This paper is structured as follows: In Sec. II we outline the necessary theoretical background, including the definition of the van Hove function, the Vineyard approximation, DDFT, and the static test particle limit. Most of this section may be safely skipped by expert readers. In Sec. III the dynamical test particle limit is introduced. Section IV summarises the model used and describes the simulation details. In Sec. V we describe results from the different dynamical approaches, the corresponding equilibrium approaches, and the free energy landscape. In Sec. VI we make some concluding remarks. Appendix A presents an exact solution of a corresponding equilibrium situation.

\section{BACKGROUND}

\section{A. The van Hove function}

We first recall the definition of the van Hove function and some of its properties; for a more detailed account see Refs. 1 and 2. Consider a set of $N$ particles with time dependent position coordinates $\mathbf{r}_{i}(t)$, where $i=1, \ldots, N$ is the particle index, and $t$ is time. The van Hove correlation function is defined as the probability of finding a particle at position $\mathbf{r}$ 
at time $t$, given that there was a particle at the origin at time $t=0$

$$
G(r, t)=\frac{1}{N}\left\langle\sum_{i=1}^{N} \sum_{j=1}^{N} \delta\left(\mathbf{r}+\mathbf{r}_{j}(0)-\mathbf{r}_{i}(t)\right)\right\rangle,
$$

where $\langle\cdot\rangle$ represents an ensemble average and $\delta(\cdot)$ is the three-dimensional Dirac delta function. $G(r, t)$ can be naturally separated into two terms, conventionally referred to as its "self" and "distinct" part, by discriminating between the cases $i=j$ and $i \neq j$, respectively. So

$$
\begin{aligned}
G(r, t)= & \frac{1}{N}\left\langle\sum_{i=1}^{N} \delta\left(\mathbf{r}+\mathbf{r}_{i}(0)-\mathbf{r}_{i}(t)\right)\right\rangle \\
& +\frac{1}{N}\left\langle\sum_{i \neq j}^{N} \delta\left(\mathbf{r}+\mathbf{r}_{j}(0)-\mathbf{r}_{i}(t)\right)\right\rangle \\
\equiv & G_{s}(r, t)+G_{d}(r, t),
\end{aligned}
$$

where the self part, $G_{s}(r, t)$, describes the average motion of the particle that was initially at the origin, whereas the distinct part, $G_{d}(r, t)$, describes the behavior of the remaining $N-1$ particles. At $t=0$, Eq. (3) reduces to the static particle-particle autocorrelation function, which is defined as

$$
\begin{aligned}
G(\mathbf{r}, 0) & =\delta(\mathbf{r})+\frac{1}{N}\left\langle\sum_{i \neq j}^{N} \delta\left(\mathbf{r}+\mathbf{r}_{j}(0)-\mathbf{r}_{i}(0)\right)\right\rangle \\
& =\delta(\mathbf{r})+\rho g(\mathbf{r}),
\end{aligned}
$$

where $g(\mathbf{r})$ is the (static) pair distribution function. For the homogeneous bulk fluid $\rho(\mathbf{r})=\rho$; isotropy implies that the dependence is only on $r=|\mathbf{r}|$. Thus, at $t=0$ :

$$
\begin{aligned}
& G_{s}(r, 0)=\delta(\mathbf{r}) \\
& G_{d}(r, 0)=\rho g(r) .
\end{aligned}
$$

From the definitions of $G_{s}(r, t)$ and $G_{d}(r, t)$, Eq. (3), it is clear that the volume integral of these functions must be a conserved quantity for all times $t$

$$
\begin{aligned}
& \int \mathrm{d} \mathbf{r} G_{s}(r, t)=1, \\
& \int \mathrm{d} \mathbf{r} G_{d}(r, t)=N-1 .
\end{aligned}
$$

The asymptotic behavior of $G(r, t)$ in bulk in the thermodynamic limit is obtained by considering $N \rightarrow \infty$ and volume $V \rightarrow \infty$ such that $N / V=\rho$ is finite

$$
\begin{aligned}
& \lim _{r \rightarrow \infty} G_{s}(r, t)=\lim _{t \rightarrow \infty} G_{s}(r, t)=0, \\
& \lim _{r \rightarrow \infty} G_{d}(r, t)=\lim _{t \rightarrow \infty} G_{d}(r, t)=\rho .
\end{aligned}
$$

A key quantity that we use below to characterize $G_{s}(r, t)$ is its width $w(t)$ defined via

$$
(w(t))^{2}=4 \pi \int_{0}^{\infty} \mathrm{d} r r^{4} G_{s}(r, t),
$$

the second moment of $G_{s}(r, t)$. It is often convenient to consider the intermediate scattering function which is related to the van Hove function via a spatial Fourier transform,

$$
F(k, t)=\int \mathrm{d} \mathbf{r} G(r, t) \exp (-i \mathbf{k} \cdot \mathbf{r})
$$

This quantity is directly accessible in light and neutron scattering experiments. ${ }^{1}$

\section{B. Approximating $G_{s}(r, t)$}

A commonly used approximation for the self part of the van Hove function is to assume a Gaussian shape: ${ }^{1}$

$$
G_{s}(r, t)=\frac{1}{\pi^{3 / 2} W(t)^{3}} \exp \left(-\frac{r^{2}}{W(t)^{2}}\right),
$$

where the width $W(t)=\sqrt{\frac{3}{2}} w(t)$, when $w(t)$ is calculated via Eq. (11). The form (13) is exact in the limits $t \rightarrow 0$ and $t \rightarrow \infty$ for all densities when the system is fluid. ${ }^{1}$ It is also exact for all times $t$ in the low density limit $\rho \rightarrow 0$, where interactions between the particles can be neglected. There are a number of approximations for $W(t)$. In molecular dynamics, at very short times $t \ll \tau_{c}$, where $\tau_{c}$ is the mean collision time, particles in a fluid do not experience collisions and therefore move freely at a constant velocity. This is akin to an ideal gas where the particle velocities follow a simple Maxwellian (Gaussian) distribution, giving

$$
W(t)=t \sqrt{2 / \beta m},
$$

where $m$ is the particle mass. Over longer times $t \gg \tau_{c}$ the particles in the fluid undergo many collisions with neighboring particles, so that the trajectory of a given particle is a random walk and thus its probability distribution $G_{s}(r, t)$ is the solution of the diffusion equation

$$
\frac{\partial G_{s}(\mathbf{r}, t)}{\partial t}=D_{l} \nabla^{2} G_{s}(\mathbf{r}, t)
$$

where $D_{l}$ is the long time self-diffusion coefficient. For the Dirac delta initial condition (5), the solution of Eq. (15) corresponds to the Gaussian form (13), with

$$
W(t)=2 \sqrt{D_{l} t} .
$$

For colloidal particles, the collisions with the solvent atoms happen so frequently that the time scale $\tau_{c}$ is much smaller than all other time scales relevant for the dynamics, such as the Brownian time scale $\tau_{B}$ which is roughly the time for a particle to travel a distance equal to its own diameter, and also the typical time scale between collisions of pairs of colloids, $\tau_{c o l}$. This means that we may set $\tau_{c} \rightarrow 0$ and that for a low density suspension of colloids Eq. (16) holds for all times $t$. Thus, we may combine Eqs. (13) and (16) to obtain

$$
G_{s}(r, t)=\left(4 \pi D_{l} t\right)^{-3 / 2} \exp \left(-\frac{r^{2}}{4 D_{l} t}\right) .
$$

We find below that for Brownian hard spheres this approximation is not only reliable in the low density limit, but is also fairly good up to intermediate densities $\rho \sigma^{3} \lesssim 0.6$. 


\section{Vineyard approximation for $G_{d}(r, t)$}

Vineyard ${ }^{25}$ suggested that one may rewrite the distinct part of the van Hove function as

$$
G_{d}(r, t)=\int \mathrm{d} \mathbf{r}^{\prime} g\left(\mathbf{r}^{\prime}\right) H\left(\mathbf{r}, \mathbf{r}^{\prime}, t\right),
$$

which is merely a redefinition of $G_{d}(r, t)$ in terms of the unknown function $H\left(\mathbf{r}, \mathbf{r}^{\prime}, t\right)$, which is the probability that if there was a particle at the origin at time $t=0$ and a second particle located at $\mathbf{r}^{\prime}$, this second particle is later located at $\mathbf{r}$ at time $t$. Vineyard's approximation is to replace $H\left(\mathbf{r}, \mathbf{r}^{\prime}, t\right)$ by $G_{s}\left(\mathbf{r}-\mathbf{r}^{\prime}, t\right)$, giving

$$
G_{d}(r, t)=\int \mathrm{d} \mathbf{r}^{\prime} g\left(\mathbf{r}^{\prime}\right) G_{s}\left(\mathbf{r}-\mathbf{r}^{\prime}, t\right) .
$$

Some comments in the literature state that the Vineyard approximation ignores important correlations that inhibit the rate at which the structure of the liquid breaks up and it therefore predicts too rapid decay of this structure. ${ }^{26}$ This may indeed be the case for fluids undergoing molecular dynamics, but for the system with Brownian dynamics (over damped stochastic equations of motion) that we consider here, we find that taking Eq. (17) together with Eq. (19) is actually fairly reliable-in particular when the fluid is at low and intermediate densities $\rho \sigma^{3} \lesssim 0.6$. We will henceforth refer to Eqs. (17) and (19) as the "Vineyard approximation" for the van Hove function.

\section{DDFT and equilibrium DFT}

The dynamics of a system of $N$ Brownian (colloidal) particles with positions $\mathbf{r}_{i}(t)$ can be modeled with the following set of (over-damped) stochastic equations of motion: ${ }^{27}$

$$
\Gamma^{-1} \frac{\mathrm{d} \mathbf{r}_{i}(t)}{\mathrm{d} t}=-\nabla_{i} U_{N}\left(\mathbf{r}^{N}, t\right)+\zeta_{i}(t)
$$

where $\mathbf{r}^{N}=\left\{\mathbf{r}_{i} ; i=1, \ldots, N\right\}$ is the set of particle coordinates, $\Gamma^{-1}$ is a friction constant characterizing the one-body drag of the solvent on the particles, $\zeta_{i}(t)$ is a stochastic white noise term and the total inter particle potential energy is

$$
U_{N}\left(\mathbf{r}^{N}, t\right)=\sum_{i=1}^{N} u\left(\mathbf{r}_{i}, t\right)+\frac{1}{2} \sum_{i=1}^{N} \sum_{j \neq i} v\left(\left|\mathbf{r}_{i}-\mathbf{r}_{j}\right|\right),
$$

which is composed of a one-body contribution due to the external potential $u$ (which may or may not be time dependent), and a sum of contributions from the pair interactions between the particles. The time evolution of the probability density for the particle coordinates $P^{(N)}\left(\mathbf{r}^{N}, t\right)$ is described by the Smoluchowski equation: ${ }^{23,27}$

$$
\frac{\partial P^{(N)}}{\partial t}=\Gamma \sum_{i=1}^{N} \nabla_{i} \cdot\left[k_{B} T \nabla_{i} P^{(N)}+\nabla_{i} U_{N} P^{(N)}\right] .
$$

The one-body density is obtained by integrating over the position coordinates of all but one particle

$$
\rho\left(\mathbf{r}_{1}, t\right)=N \int \mathrm{d} \mathbf{r}_{2} \ldots \int \mathrm{d} \mathbf{r}_{N} P\left(\mathbf{r}^{N}, t\right) .
$$

Integrating the Smoluchowski Eq. (22) we obtain the key equation of DDFT: ${ }^{23}$

$$
\frac{\partial \rho(\mathbf{r}, t)}{\partial t}=\Gamma \nabla \cdot\left[\rho(\mathbf{r}, t) \nabla \frac{\delta F[\rho(\mathbf{r}, t)]}{\delta \rho(\mathbf{r}, t)}\right],
$$

where $F[\rho]$ is taken to be the equilibrium total Helmholtz free energy functional

$$
\begin{array}{r}
F[\rho(\mathbf{r})]=k_{B} T \int \mathrm{d} \mathbf{r} \rho(\mathbf{r})\left[\ln \left(\Lambda^{3} \rho(\mathbf{r})\right)-1\right] \\
+F_{\mathrm{ex}}[\rho(\mathbf{r})]+\int \mathrm{d} \mathbf{r} u(\mathbf{r}) \rho(\mathbf{r}),
\end{array}
$$

where the first term on the right hand side is the ideal-gas contribution to the free energy, $\Lambda$ is the thermal de Broglie wavelength, $F_{\text {ex }}[\rho(\mathbf{r})]$ is the excess (over ideal gas) part of the free energy, which is in general unknown exactly, and we have suppressed the dependence on temperature $T$ and volume $V$ in the notation. In obtaining Eq. (24) we have made the approximation that equal-time two-body correlations at each time $t$ in the non equilibrium situation are the same as those of an equilibrium fluid with the same one-body density profile $\rho(\mathbf{r}, t)$, generated by an appropriate external potential. ${ }^{21-23}$ It has been shown in a variety of cases that the DDFT (24) is reliable in predicting the time-evolution of $\rho(\mathbf{r}, t)$, when solved in conjunction with a sufficiently accurate approximation for the equilibrium Helmholtz free energy functional $F[\rho(\mathbf{r})]$ - see for example the results presented in Refs. 21, 28-35, and 36.

Although in the following we will not go beyond dynamics that are local in time, it is worth mentioning that more generally, going beyond the case of particles with stochastic over damped equations of motion (20), Chan and Finken ${ }^{37}$ established a rigorous DDFT for classical fluids showing that the time evolution of the one body density $\rho(\mathbf{r}, t)$ is obtained from the solution of

$$
\begin{aligned}
& \frac{\partial \rho(\mathbf{r}, t)}{\partial t}=-\nabla \cdot \mathbf{j}(\mathbf{r}, t), \\
& \frac{\partial \mathbf{j}(\mathbf{r}, t)}{\partial t}=\mathbf{P}[\rho(\mathbf{r}, t)],
\end{aligned}
$$

where $\mathbf{j}(r, t)$ is the particle current density, and Eq. (26) represents a continuity equation for the one-body density $\rho(\mathbf{r}, t)$. One should, of course, expect on general grounds for the dynamical equations to be of this form ${ }^{38-40}$-recall that Eq. (27) is the continuity equation. However, the functional $\mathbf{P}[\rho(\mathbf{r}, t)]$ that governs the time evolution of $\rho(\mathbf{r}, t)$ takes a form that depends on the equations of motion of the particles-i.e., it depends on whether the particles evolve under Newtonian dynamics or have stochastic equations of motion such as Eq. (20).

Due to the fact that in general the functional $\mathbf{P}[\rho(\mathbf{r}, t)]$ in Eq. (27) is an unknown quantity, one is prevented from applying our DDFT approach for calculating dynamic correlation functions, and we are restricted to the Brownian case (20) outlined above. The particular approximation used in Eqs. (26) and (27) to obtain Eq. (24) is to assume the one particle current density to be of the form

$$
\mathbf{j}(\mathbf{r}, t)=-\Gamma \rho(\mathbf{r}, t) \nabla \frac{\delta F[\rho(\mathbf{r}, t)]}{\delta \rho(\mathbf{r}, t)} .
$$


Nevertheless, there is much active research aimed at going beyond the simple overdamped case. ${ }^{41-46}$

In what follows we will relate the van Hove function to the time evolution of the one-body density profiles of a binary mixture; we therefore require the multicomponent generalization $^{30}$ of Eq. (24):

$$
\frac{\partial \rho_{i}(\mathbf{r}, t)}{\partial t}=\Gamma \nabla \cdot\left[\rho_{i}(\mathbf{r}, t) \nabla \frac{\delta F\left[\left\{\rho_{i}\right\}\right]}{\delta \rho_{i}(\mathbf{r}, t)}\right],
$$

where $F\left[\left\{\rho_{i}\right\}\right]$ has the following form [cf. Eq. (25)]: ${ }^{47}$

$$
\begin{aligned}
F\left[\left\{\rho_{i}\right\}\right]= & k_{B} T \sum_{i} \int \mathrm{d} \mathbf{r} \rho_{i}(\mathbf{r})\left[\ln \Lambda^{3} \rho_{i}(\mathbf{r})-1\right] \\
& +F_{\mathrm{e} x}\left[\left\{\rho_{i}\right\}\right]+\sum_{i} \int \mathrm{d} \mathbf{r} u_{i}(\mathbf{r}) \rho_{i}(\mathbf{r}),
\end{aligned}
$$

where the summations run over all species $i$. Given an initial set of density profiles, $\left\{\rho_{i}(\mathbf{r}, t=0)\right\}$, we may employ the DDFT Eqs. (29) and (30) to calculate the full time evolution of the one-body density profiles $\rho_{i}(\mathbf{r}, t)$.

For completeness, we recall some of the key results from equilibrium. $^{1,20}$ For a given set of (one-body) external potentials $\left\{u_{i}(\mathbf{r})\right\}$, the unique set of one-body density profiles $\left\{\rho_{i}(\mathbf{r})\right\}$ are those which minimize the Helmholtz free energy of the system $F\left[\left\{\rho_{i}\right\}\right]$, subject to the constraint that the average number of particles of each species $\int \mathrm{d} \mathbf{r} \rho_{i}(\mathbf{r})=N_{i}$ is fixed. This is equivalent to an unconstrained minimization of the grand potential functional

$$
\Omega\left[\left\{\rho_{i}\right\}\right]=F\left[\left\{\rho_{i}\right\}\right]-\sum_{i} \mu_{i} \int \mathrm{d} \mathbf{r} \rho_{i}(\mathbf{r}),
$$

where the Lagrange multiplier $\mu_{i}$ is the chemical potential of species $i$. Minimization with respect to variations in the density profiles yields the following set of the Euler-Lagrange equations: 1,20

$$
\frac{\delta F\left[\left\{\rho_{i}\right\}\right]}{\delta \rho_{i}(r)}=\mu_{i}
$$

The Euler-Lagrange equations can be rewritten as

$$
\rho_{i}(\mathbf{r})=\Lambda^{-3} \exp \left[\beta \mu_{i}-\beta u_{i}(\mathbf{r})+c_{i}^{(1)}\left(\mathbf{r}\left[\left\{\rho_{j}\right\}\right]\right)\right],
$$

where

$$
c_{i}^{(1)}\left(\mathbf{r} ;\left[\left\{\rho_{j}\right\}\right]\right)=-\beta \frac{\delta F_{\mathrm{ex}}\left[\left\{\rho_{j}\right\}\right]}{\delta \rho_{i}(\mathbf{r})},
$$

is the one-body direct correlation functional. The set of density profiles that satisfy Eq. (33) minimize the free energy and are the equilibrium density profiles. When the equilibrium set of profiles $\left\{\rho_{i}(\mathbf{r})\right\}$ are substituted into Eq. (31), the grand potential $\Omega$ of the system is obtained.

\section{E. Percus' test particle limit}

Here we give a derivation of Percus' (static) test particle limit closely following Ref. 48. Consider a one component system such that the Helmholtz free energy, $F$, is given by Eq. (25). We are interested in the change in $\rho(\mathbf{r})$ when the external potential is changed from the potential $u^{\prime}(\mathbf{r})$ to the potential $u(\mathbf{r})$. To this end we perform a functional Taylor expansion of $F_{\mathrm{ex}}[\rho]$ in powers of $\Delta \rho(\mathbf{r})=\rho(\mathbf{r})-\rho^{\prime}(\mathbf{r})$.
For the sake of simplicity we consider the change in going from $u^{\prime}(\mathbf{r})=0$ to a spherically symmetric external potential $u(r)$. The variable in the Taylor expansion is then $\Delta \rho(r)=\rho(r)-\rho^{b}$, where $\rho^{b}$ is the bulk density and the expansion of $F_{\mathrm{ex}}[\rho]$ to second order in $\Delta \rho(r)$ is

$$
\begin{aligned}
F_{\mathrm{ex}}[\rho]= & F_{\mathrm{ex}}\left[\rho^{b}\right]+\left.\int \mathrm{d} \mathbf{r} \frac{\delta F_{\mathrm{ex}}[\rho]}{\delta \rho(r)}\right|_{\rho^{b}} \Delta \rho(r) \\
& +\left.\frac{1}{2} \int \mathrm{d} \mathbf{r} \int \mathrm{d} \mathbf{r}^{\prime} \frac{\delta^{2} F_{\mathrm{ex}}[\rho]}{\delta \rho(r) \delta \rho\left(r^{\prime}\right)}\right|_{\rho^{b}} \Delta \rho(r) \Delta \rho\left(r^{\prime}\right) \\
& +O\left(\Delta \rho^{3}\right) .
\end{aligned}
$$

Although the form of $F_{\mathrm{ex}}$ is not specified, the functional derivatives are related to identifiable properties of the system, so that evaluating them at $\rho(r)=\rho^{b}$ gives

$$
\begin{aligned}
\left.\frac{\delta F_{\mathrm{ex}}[\rho]}{\delta \rho(r)}\right|_{\rho^{b}} & =-k_{B} T \mu_{\mathrm{ex}}, \\
\left.\frac{\delta^{2} F_{\mathrm{ex}}[\rho]}{\delta \rho(r) \delta \rho\left(r^{\prime}\right)}\right|_{\rho^{b}} & =-k_{B} T c^{(2)}\left(\left|\mathbf{r}-\mathbf{r}^{\prime}\right|\right), \\
\frac{\delta O\left(\Delta \rho^{3}\right)}{\delta \rho(r)} & =B(r),
\end{aligned}
$$

where $\mu_{\mathrm{ex}}$ is the excess chemical potential, $c^{(2)}(r)$ is the (pair) direct correlation function, and $B(r)$ is an unknown function that contains the higher order terms of the Taylor expansion. Substituting Eqs. (35) and (36) into Eq. (25), and then minimizing the functional with respect to variations in $\rho(r)$, we obtain the following Euler-Lagrange equation [cf. Eq. (32)]:

$$
\frac{\delta F}{\delta \rho(r)}=\mu=k_{B} T \ln \left(\Lambda^{3} \rho^{b}\right)+\mu_{\mathrm{ex}},
$$

where we have separated the chemical potential $\mu$ into an ideal gas and an excess (over ideal gas) contribution $\mu_{\mathrm{ex}}$. In the case of a spherically symmetric external potential, Eq. (37) may be rewritten as

$$
\frac{\rho(r)}{\rho^{b}}=\exp \left[-\beta u(r)+\int \mathrm{d} \mathbf{r}^{\prime} c\left(\left|r-r^{\prime}\right|\right) \Delta \rho\left(r^{\prime}\right)+B(r)\right] \text {. }
$$

For the same one-component system the bulk OrnsteinZernike (OZ) equation for the total correlation function, $h(r)=g(r)-1$, reads as follows:

$$
h(r)=c(r)+\rho^{b} \int \mathrm{d} \mathbf{r}^{\prime} h\left(r^{\prime}\right) c\left(\left|r-r^{\prime}\right|\right) .
$$

It can be shown through diagrammatic methods ${ }^{1}$ that the $\mathrm{OZ}$ equation has the general solution

$$
h(r)=c(r)+\ln (g(r))+\beta v(r)+b(r),
$$

where $v(r)$ is the inter particle pair potential, and $b(r)$ is the bridge function composed of the sum of all the so-called "bridge" diagrams. ${ }^{1}$ Substituting Eq. (40) into Eq. (39) we obtain

$$
g(r)=\exp \left[-\beta v(r)+\int \mathrm{d} \mathbf{r}^{\prime} c\left(\left|r-r^{\prime}\right|\right) \rho^{b} h\left(r^{\prime}\right)+b(r)\right]
$$


If we compare Eqs. (38) and (41) we find they have the same structure, and that they may be formally identified. If we set $u(r)=v(r)$ in Eq. (38), it can be shown through diagrammatic methods ${ }^{1,49}$ that $b(r)=B(r)$ and that

$$
g(r)=\rho(r) / \rho^{b},
$$

or alternatively, $\rho h(r)=\Delta \rho(r)$. Thus when $u(r)=v(r)$, Eqs. (38) and (41) become identical. Therefore we note that not only can the OZ relationship be derived from the free energy functional, ${ }^{20}$ but also that the equilibrium one-body density profile in the presence of an external potential $u(r)=v(r)$ is related to the (two-body) radial distribution function via Eq. (42). We should recall at this point that although many formal statements can be made about the bridge function $b(r)$, in practice it is an unknown function, and all theories for $g(r)$ constitute some form of approximation for $b(r) .{ }^{1}$ For example, if we set $b(r)=B(r)=0$ then Eq. (38) is equivalent to using the hyper netted chain (HNC) approximation ${ }^{1}$ to the $\mathrm{OZ}$ equation. Furthermore, Percus ${ }^{19}$ showed that by Taylor expanding with different functions of $\rho(r)$ one may retrieve the Percus-Yevick and other closures to the OZ equations. This result may also be generalized to inhomogeneous systems. ${ }^{48}$

\section{F. Zero-dimensionality route to $g(r)$}

We present an alternative method for calculating $g(r)$, although its basis is the same key idea that underpins Percus' test particle limit described above: that $g(r)$ can be obtained from the density profile of a fluid around a fixed test particle. The key difference is that instead of treating the test particle as a fixed external potential, in the zero-dimensionality route we treat the test particle via its density distribution. The density profile of a particle fixed at a point (i.e., in zero-dimensional space-hence our choice of name for this limit) takes the form of a Dirac delta function. Having fixed this contribution to the density distribution [cf. Eq. (4)], one can then calculate the density distribution of the remaining particles in the presence of the test particle. Specifically, we can write the grand potential functional as

$$
\begin{aligned}
\Omega^{*}[\rho g(r)]= & \left.F_{\mathrm{id}}[\rho g(r)]+F_{\mathrm{ex}}[\delta(\mathbf{r})+\rho g(r))\right] \\
& -\mu \int \mathrm{d} \mathbf{r} \rho g(r),
\end{aligned}
$$

where $\rho g(r)$ is the density distribution of the remaining particles-the quantity we wish to calculate. Note that here, and in what follows, $\rho$ is the bulk density. The ideal gas term $F_{\text {id }}$ does not contain the Dirac delta contribution-we have crossed over to a system with $N-1$ particles. Since the bulk fluid density $\rho$ is necessarily fixed, we must simply minimize $\Omega^{*}$ with respect to variations in $g(r)$, giving the following Euler-Lagrange equation to be solved for $g(r)$ :

$$
\frac{\delta \Omega^{*}}{\delta g(r)}=0 .
$$

An alternative means of calculating $g(r)$ is to treat the system as a binary mixture. The test particle (which we label " $s$ "), with density distribution $\rho_{s}(r)=\delta(\mathbf{r})$, is one species and then we use the DFT for a binary mixture to calculate the density profile of the remain particles $\rho_{d}(r)$ in the presence of the density profile $\rho_{s}(r)$ for the fixed particle, treating the remaining particles as a second species " $d$ " in the mixture. $\rho_{d}(r)$ is the solution of

$$
\frac{\delta \Omega^{\dagger}}{\delta \rho_{d}(r)}=0,
$$

where $\Omega^{\dagger}$ is a modified version of Eq. (31) where $\rho_{s}(r)=\delta(\mathbf{r})$ is fixed and therefore the ideal Helmholtz free energy, $F_{\text {id }}\left[\rho_{d}\right]$, does not depend on $\rho_{s}(\mathbf{r})$, cf. Eq. (43).

When using an approximate free energy functional, there is a difference between the zero-dimensional limit and Percus' limit for calculating $g(r)$. This is because in the zerodimensionality limit, in contrast to Percus' method, the test particle at the origin does not interact with the fluid via an external potential $u(r)=v(r)$, that is identical to the pair potential, but rather via an approximation $u^{*}(r)$ to the pair potential, generated by the approximate density functional. We calculate below in Sec. IV C an explicit expression for $u^{*}(r)$ in the case of hard spheres treated using the RY approximation for the free energy. We will also discuss further the relation between Percus' test particle limit and the zero-dimensionality limit.

\section{DYNAMIC TEST PARTICLE LIMIT}

\section{A. Definition}

We next extend the static test particle limit and consider the dynamical situation which allows us to calculate the van Hove function $G(r, t)$. The key is the following observation: consider a fixed test particle of species " $s$ " (self) located at the origin; due to Percus we know that in this situation the density distribution of the remaining particles $\rho(r)=\rho g(r)$. Now consider releasing the test particle at time $t=0$ and allowing it to move through the fluid. When this happens its probability (density) distribution $\rho_{s}(r, t)$ changes from a Dirac delta function (at $t=0$ ) to a distribution with a non zero value for some points away from the origin. If we now recall the definition of the function $G_{s}(r, t)$ in Eq. (3), we see that $G_{s}(r, t)$ gives the probability that a particle initially located at the origin has moved a distance $r$ away from the origin after time $t$. Therefore, in this situation, $\rho_{s}(r, t) \equiv G_{s}(r, t)$ for all times $t \geq 0$. Similarly, if we consider how the remaining particles redistribute themselves as the test particle moves away from the origin, we see from Eq. (3) that the probability of finding any one of these particles a distance $r$ from the origin at time $t$ is given by $G_{d}(r, t)$. We label the remaining particles as being particles of species " $d$ " (distinct) and having the density profile $\rho_{d}(r, t)$. Thus, as in the static test particle case, we may connect the two parts of the van Hove function with the density profiles of a two-component system of species $s$ and $d$,

$$
\begin{gathered}
G_{s}(r, t) \equiv \rho_{s}(r, t), \\
G_{d}(r, t) \equiv \rho_{d}(r, t),
\end{gathered}
$$

where species $s$ is composed of only one particle, the test particle, and $\int \mathrm{d} \mathbf{r} \rho_{s}(r, t)=1$, so that Eq. (7) is satisfied. We may therefore formally set the pair potential for interactions between species $s$ particles $v_{s s}(r)=0$. The density profile for 
species $d$ must satisfy the normalization constraint (8), and the self-distinct and distinct-distinct pair potentials must be identical, $v_{s d}(r)=v_{d d}(r)=v(r)$. This is equivalent to modeling a one-component system, but treating one particle separately from the rest. Recall that at time $t=0$ we know the test particle's position exactly from Eq. (5) and combining this with Eqs. (6), (42), and (46) we obtain

$$
\begin{gathered}
G_{s}(r, t=0) \equiv \rho_{s}(r, t=0)=\delta(r), \\
G_{d}(r, t=0) \equiv \rho_{d}(r, t=0)=\rho g(r) .
\end{gathered}
$$

The connections made in Eq. (46) between the self and distinct parts of the van Hove function and the density profiles $\rho_{s}(r, t)$ and $\rho_{d}(r, t)$ in the dynamical test particle limit described above are conceptually important. However, we have merely shifted the problem of how to determine $G(r, t)$ onto the problem of how to determine the time evolution of the two coupled density profiles $\rho_{s}(r, t)$ and $\rho_{d}(r, t)$. The solution that we use in this paper is to use DDFT, i.e., Eq. (29) are integrated forward in time with Eqs. (47) providing the initial time, $t=0$, density profiles. The resulting time series of density profiles gives the self and distinct parts of the van Hove function through Eq. (46). Henceforth, we refer to this as the "dynamical test particle" theory.

\section{B. Approximate solution}

Before proceeding to the results that we obtain from following the calculation scheme described above, it is worth examining an approximate analytical solution that may be obtained as follows: from Eqs. (29), (30), and (34) we may write the DDFT equations for the two density profiles $\rho_{s}(r, t)$ and $\rho_{d}(r, t)$ as

$$
\frac{\partial \rho_{i}(\mathbf{r}, t)}{\partial t}=D \nabla^{2} \rho_{i}(\mathbf{r}, t)+\Gamma \nabla \cdot\left[\rho_{i}(\mathbf{r}, t) \nabla c_{i}^{(1)}(\mathbf{r}, t)\right],
$$

where $i=s, d$ and the diffusion coefficient $D=k_{B} T \Gamma$. If we set the second term on the right hand side to zero and we set $D=D_{l}$ then we obtain Eq. (15) for $\rho_{s}(r, t)=G_{s}(r, t)$ and thus the solution to the DDFT for the self part of the van Hove function in this limit is the Gaussian form in Eq. (17). Similarly, for species $d$, when we assume that the second term on the right hand side of Eq. (48) can be neglected, then we obtain

$$
\frac{\partial \rho_{d}(\mathbf{r}, t)}{\partial t}=D \nabla^{2} \rho_{d}(\mathbf{r}, t)
$$

If we now assume the Vineyard approximation

$$
\rho_{d}(r, t)=\int \mathrm{d} \mathbf{r}^{\prime} g\left(\mathbf{r}^{\prime}\right) \rho_{s}\left(\left|\mathbf{r}-\mathbf{r}^{\prime}\right|, t\right)
$$

[cf. Eq. (19)], then after Fourier transforming Eq. (49), together with Eq. (50) we obtain

$$
\hat{g}(k) \frac{\partial \hat{\rho}_{d}(k, t)}{\partial t}=-k^{2} D \hat{g}(k) \hat{\rho}_{d}(k, t),
$$

where $\hat{g}(k)$ is the Fourier transform of the radial distribution function $g(r)$ and $\hat{\rho}_{d}(k, t)$ is the Fourier transform of $\rho_{d}(r, t)$. Dividing both sides of Eq. (51) by $\hat{g}(k)$ and then taking the inverse Fourier transform we obtain Eq. (15) for $\rho_{s}(r, t)=G_{s}(r, t)$. Thus the Gaussian form in Eq. (17) together with the Vineyard approximation (50) for $\rho_{d}(r, t)$, together form a self-consistent solution to the DDFT equations in the dynamical test particle limit, in the case where we can neglect the contribution from the second term on the righthand side of Eq. (48). This term is zero in the ideal-gas limit when the excess contribution to the free energy $F_{\mathrm{ex}}=0$, in Eq. (30), or when $\rho \rightarrow 0$. However, we find below for hard spheres that this approximation is reliable well beyond the ideal gas regime, which suggests that in the test particle limit $c_{s}^{(1)}$ and $c_{d}^{(1)}$ in Eq. (48) must both be slowly varying (almost constant) functions, so that their gradients are small.

\section{MODEL FOR HARD SPHERES}

\section{A. Simulation method}

In order to provide benchmark results, we calculate the van Hove function by integrating the equations of motion (20) using standard BD computer simulations. ${ }^{50}$ In order to apply the algorithm we model the hard spheres with a steep continuous pair potential

$$
\beta v(r)= \begin{cases}(\sigma / r)^{36}-1 & r<\sigma, \\ 0 & \text { otherwise }\end{cases}
$$

We solve Eq. (20) using the Euler forward algorithm using a time step $\delta t=1 \times 10^{-5} \tau_{B}$; recall that the Brownian time $\tau_{B}=\sigma^{2} / D$, where $D=\Gamma k_{B} T$ is the Stokes-Einstein diffusion coefficient. The random forces $\zeta_{i}$ in Eq. (20) mimic the interaction between particles and solvent, and are sampled from a Gaussian distribution with zero mean and variance $2 D \delta t$. The simulations are carried out using $N=1728$ particles at densities $\rho \sigma^{3}=N(\sigma / L)^{3}=0.2,0.4,0.6,0.8$, and 1 in a cubic box of volume $L^{3}$.

After an equilibration time of $50 \tau_{B}$, we sampled the distribution functions $G_{s}(r, t)$ and $G_{d}(r, t)$ at the times $t / \tau_{B}=$ $0.01,0.1$, and 1 . The distribution functions are averaged over all possible time intervals $t / \tau_{B}$ of a single simulation run. The total simulated times are $2 \tau_{B}, 20 \tau_{B}$, and $200 \tau_{B}$ for the short, medium, and long time intervals, respectively. The scaled intermediate scattering function is calculated from the density autocorrelation function in Fourier space, $\phi(k, t)=\left\langle n_{k}(t) n_{-k}(0)\right\rangle /\left\langle n_{k}(0) n_{-k}(0)\right\rangle$, where $n_{k}(t)=\sum_{i=1}^{N}$ $\exp \left(-i \mathbf{k} \cdot \mathbf{r}_{i}(t)\right)$ are the Fourier components of the local number density.

\section{B. The excess free energy functional}

In order to implement the dynamical test particle limit we must (as is almost always the case in density functional theory calculations) select an approximation for the excess part of the Helmholtz free energy functional, Eq. (30). We use the RY functional, ${ }^{24}$ which is obtained from the two-component generalization of Eqs. (35) and (36) by neglecting all terms of order $O\left(\Delta \rho^{3}\right)$ and higher. We obtain

$$
\begin{aligned}
& F_{\mathrm{ex}}\left[\rho_{s}, \rho_{d}\right] \\
& \quad=V f_{\mathrm{ex}}(\rho)+f_{\mathrm{ex}}^{\prime}(\rho)\left\{\int \mathrm{d} \mathbf{r}\left(\rho_{d}(\mathbf{r})-\rho\right)+\int \mathrm{d} \mathbf{r} \rho_{s}(\mathbf{r})\right\}
\end{aligned}
$$




$$
\begin{aligned}
& -\frac{1}{2} \int \mathrm{d} \mathbf{r} \int \mathrm{d} \mathbf{r}^{\prime} c\left(\left|\mathbf{r}-\mathbf{r}^{\prime}\right|\right)\left\{\left(\rho_{d}(\mathbf{r})-\rho\right)\left(\rho_{d}\left(\mathbf{r}^{\prime}\right)-\rho\right)\right. \\
& \left.+\left(\rho_{d}(\mathbf{r})-\rho\right) \rho_{s}\left(\mathbf{r}^{\prime}\right)+\rho_{s}(\mathbf{r})\left(\rho_{d}\left(\mathbf{r}^{\prime}\right)-\rho\right)\right\},
\end{aligned}
$$

where $f_{\mathrm{ex}}(\rho)$ is the bulk excess free energy per unit volume, $V$ is the volume of the system, $f_{\mathrm{ex}}^{\prime}=\partial f_{\mathrm{ex}} / \partial \rho$ and $c(r)$ is the bulk pair direct correlation function of the hard sphere fluid with bulk density $\rho$. We use $f_{\text {ex }}$ and $c(r)$ as given by the PercusYevick approximation. ${ }^{1}$

Equation (53) is perhaps the simplest functional that one could use to calculate hard sphere fluid density profiles. Our reasons for using this functional are: (i) The structure of the functional is relatively simple (and as a consequence is widely used within liquid state approaches). (ii) Within the RY functional it is straightforward to neglect the species $s$ intra species interactions which is necessary to ensure that $\rho_{s}(r, t)$ represents a single particle. (iii) Finally, the RY functional was the first functional to correctly reproduce freezing phenomena in hard spheres.

\section{Static structure of the fluid}

In Fig. 1 we display the radial distribution function and static structure factor for a bulk fluid of hard spheres for the densities $\rho \sigma^{3}=0.2,0.4,0.6,0.8$, and 1 . We show results
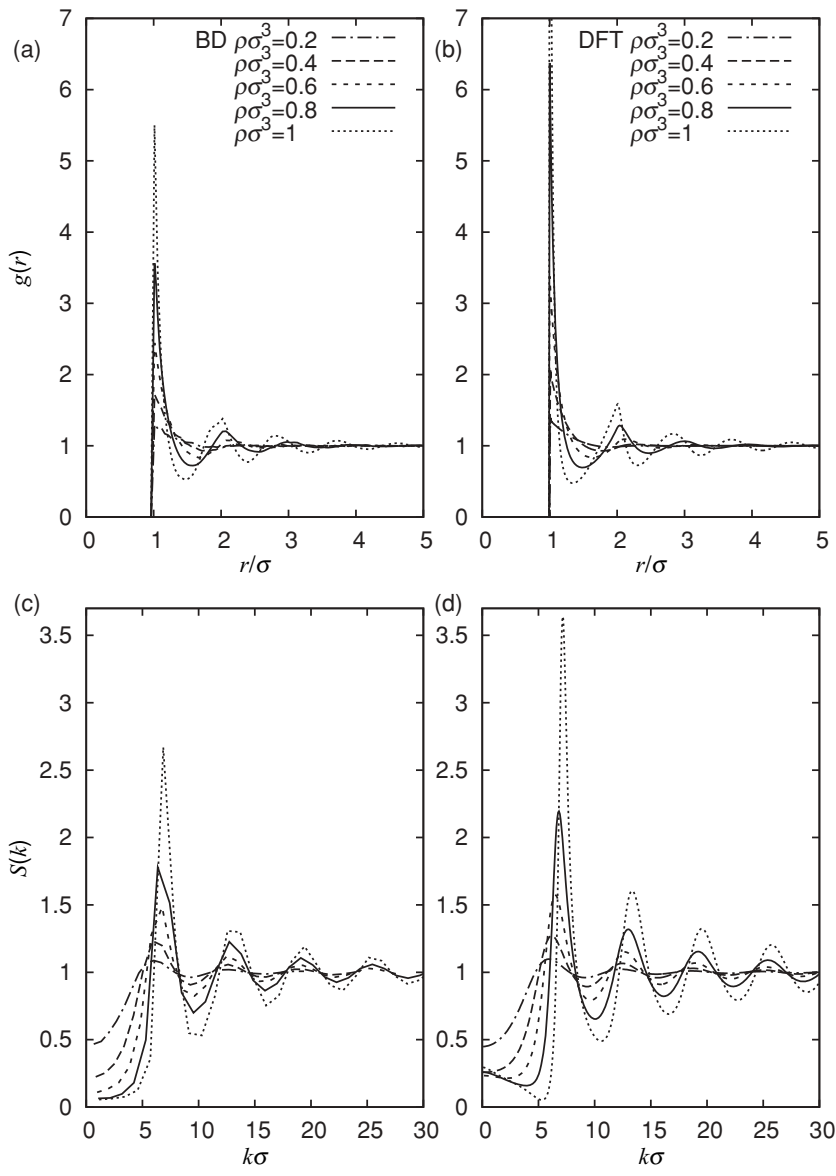

FIG. 1. (a) and (b) Display radial distribution functions, and (c) and (d) static structure factors for a bulk fluid of hard spheres at densities $\rho \sigma^{3}=0.2,0.4$, $0.6,0.8$, and 1. (a) and (c) are obtained from BD simulations and (b) and (d) via Percus' static test particle limit using the RY functional. obtained from BD simulations, together with the results from the static test particle limit using the RY approximation for the excess free energy (53). One can observe that for low and intermediate densities $\rho \sigma^{3} \lesssim 0.6$, the test particle results are in good agreement with those from the simulations. However, as the density increases, the test particle results become less reliable. We see in Fig. 1(b) that the theory overestimates the contact value, $g\left(r=\sigma^{+}\right)$. This in turn leads to the discrepancies in the static structure factor at high densities in Fig. 1(d); $S(k)$ is obtained by Fourier transforming the data in Fig. 1(b). The overall conclusion to be drawn from Fig. 1 is that the test particle method combined with the RY functional provides a reliable description of the fluid structure for low and intermediate densities, but at higher densities $\rho \sigma^{3}>0.6$, the theory is only qualitatively correct.

We return now to the discussion of the somewhat subtle issues concerning the relation between Percus' test particle limit and the zero-dimensionality limit. The results from these two calculations are not the same when one uses an approximate expression for the free energy, such as the RY functional (53). Combining Eqs. (53) and (33), we obtain the following:

$$
\begin{aligned}
\rho_{d}(r)= & \Lambda^{-3} \exp \left[\beta \mu_{d}-\beta f_{\mathrm{ex}}^{\prime}(\rho)\right. \\
& +\int \mathrm{d} \mathbf{r}^{\prime} c\left(\left|\mathbf{r}-\mathbf{r}^{\prime}\right|\right) \rho_{s}\left(r^{\prime}\right) \\
& \left.+\int \mathrm{d} \mathbf{r}^{\prime} c\left(\left|\mathbf{r}-\mathbf{r}^{\prime}\right|\right)\left(\rho_{d}\left(r^{\prime}\right)-\rho\right)\right] .
\end{aligned}
$$

Now, recall that a test-particle calculation involves fixing one of the particles at the origin, treating it as an external potential, and then determining the density profile of the fluid (species $d$ ) under the influence of this external potential. Doing this, using the RY approximation for $F_{\mathrm{ex}}\left[\rho_{s}, \rho_{d}\right]$, Eq. (53), we obtain

$$
\begin{aligned}
\rho_{d}(r)= & \Lambda^{-3} \exp \left[\beta \mu_{d}-\beta f_{\mathrm{ex}}^{\prime}(\rho)-\beta u(r)\right. \\
& \left.+\int \mathrm{d} \mathbf{r}^{\prime} c\left(\left|\mathbf{r}-\mathbf{r}^{\prime}\right|\right)\left(\rho_{d}\left(r^{\prime}\right)-\rho\right)\right] .
\end{aligned}
$$

Comparing Eq. (54) and (55), we see that Eq. (54) is merely Eq. (55) with the external potential $\beta u(r)=\beta v(r)$ replaced by the effective potential

$$
\beta u^{*}(r)=-\int \mathrm{d} \mathbf{r}^{\prime} c\left(\left|\mathbf{r}-\mathbf{r}^{\prime}\right|\right) \rho_{s}\left(r^{\prime}\right)
$$

In the $w=0$ limit, when $\rho_{s}(r)=\delta(r)$, we then obtain

$$
\beta u^{*}(r)=-c(r) .
$$

One consequence of this random phase like approximation is that the core condition is violated. The degree to which the core condition is violated could be used as an indicator toward the reliability of any approximate free energy functional.

In the remainder of this paper we will display results and distribution functions that are derived from Percus' test particle results used as initial condition, though we will draw attention to results from the zero-dimensionality route where appropriate. 

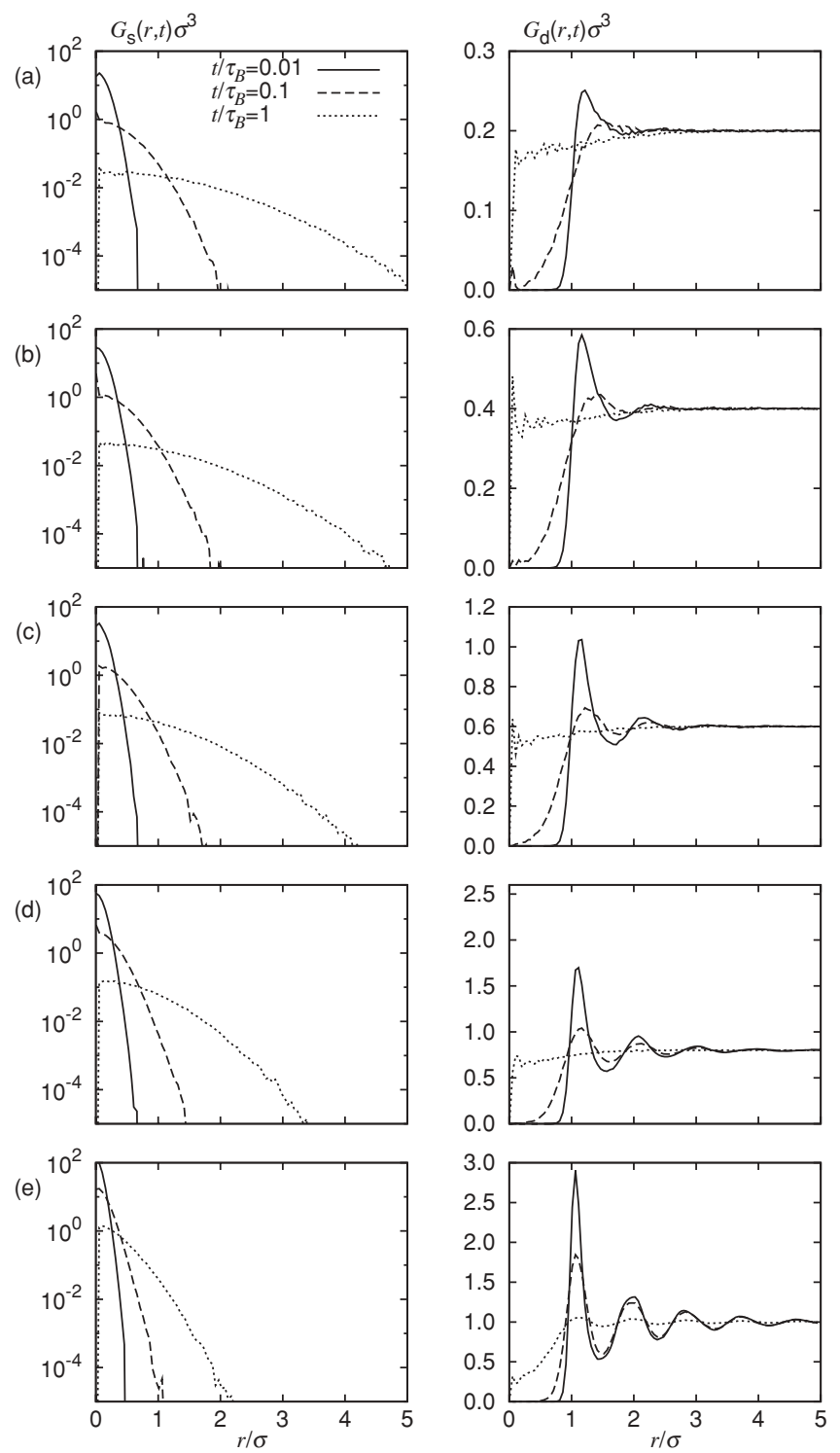

FIG. 2. The self and distinct parts of the van Hove distribution function, $G_{s}(r, t)$ and $G_{d}(r, t)$, for a hard sphere fluid, measured in BD simulations at densities: (a) $\rho \sigma^{3}=0.2$, (b) $\rho \sigma^{3}=0.4$, (c) $\rho \sigma^{3}=0.6$, (d) $\rho \sigma^{3}=0.8$, (e) $\rho \sigma^{3}=1$. The results are plotted for times $t / \tau_{B}=0.01$ (solid line), 0.1 (dashed line), and 1 (dotted line). In the semi logarithmic scale of $G_{s}(r, t)$ versus $r$ a Gaussian appears as a parabola. The $G_{d}(r, t)$ results are shown on a linear scale.

\section{RESULTS}

\section{A. Dynamic approaches}

In Fig. 2 we display the two parts of the van Hove functions, $G_{s}(r, t)$ and $G_{d}(r, t)$, measured in the BD simulations for fluid densities $\rho \sigma^{3}=0.2,0.4,0.6,0.8$, and 1 . The different curves correspond to the times $t / \tau_{B}=0.01,0.1$, and 1. $G_{s}(r, t)$ appear to have a near-Gaussian form for all times and densities. For short times $G_{d}(r, t)$ exhibits a correlation hole for $r<\sigma$ and it is highly structured for (larger) $r>\sigma$. At later times the structure in $G_{d}(r, t)$ diminishes and the correlation hole becomes "filled in". Recall that Eq. (10) defines the long time limit. Increasing the density beyond $\rho \sigma^{3}=1$, we find that the simulated system crystallizes onto a regular lattice and there is no evidence of glass forming behavior.
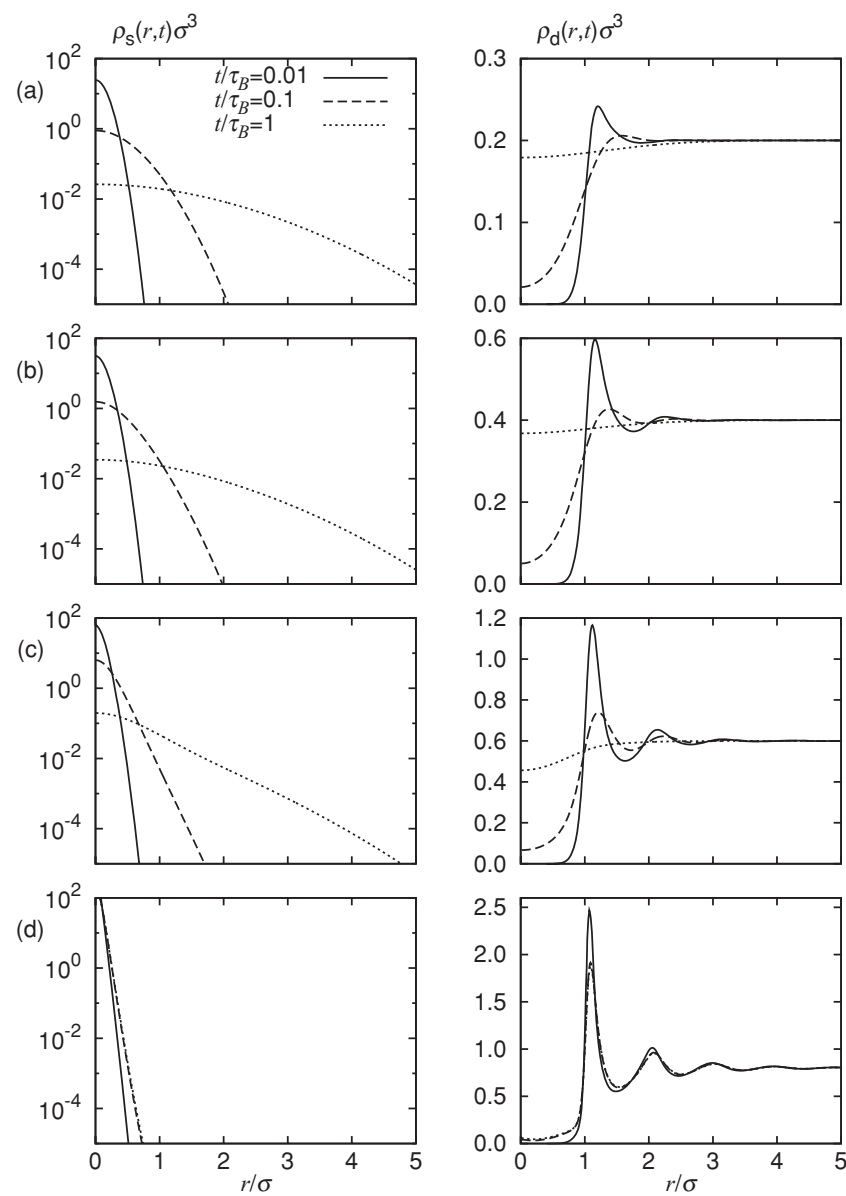

FIG. 3. The " $s$ " and " $d$ " density profiles, $\rho_{s}(r, t)$ and $\rho_{d}(r, t)$, obtained from the dynamical test particle theory, for densities: (a) $\rho \sigma^{3}=0.2$, (b) $\rho \sigma^{3}=0.4$, (c) $\rho \sigma^{3}=0.6$, (d) $\rho \sigma^{3}=0.8$. The results are plotted for times $t / \tau_{B}=0.01$ (solid line), 0.1 (dashed line), and 1 (dotted line). In (d), after a short time, the system reaches an "arrested state," where the density profiles no longer evolve in time and the width of $\rho_{s}(r, t \rightarrow \infty)$ remains finite.

In Fig. 3 we display the one-body density profiles, $\rho_{s}$ $(r, t)$ and $\rho_{d}(r, t)$, from the DDFT dynamical test particle method for bulk fluid densities $\rho \sigma^{3}=0.2,0.4,0.6$, and 0.8 . As initial condition, $\rho_{d}(r, t=0)=g(r)$, we have used Percus' test particle method for calculating $g(r)$, as shown in Fig. 1. The results in Fig. 3 correspond to the same times as the BD curves displayed in Fig. 2, namely $t / \tau_{B}=0.01,0.1$, and 1. Comparing the BD results in Fig. 2 with the DDFT results in Fig. 3 we observe that for densities $\rho \sigma^{3}=0.2,0.4$, and 0.6 , there is good qualitative agreement between the simulation and DDFT results. The $\rho_{d}(r, t)$ results show a similar amount of structure as the $G_{d}(r, t)$ results, and $\rho_{s}(r, t)$ has a very similar magnitude and range as $G_{s}(r, t)$, although for $\rho \sigma^{3}=0.6, \rho_{s}(r, t)$ shows some departures from the almost Gaussian shape observed in the simulation results, particularly at $t / \tau_{B}=1$. For $\rho \sigma^{3}=0.8$ we find that the dynamic test particle method predicts that after a short time $t / \tau_{B} \sim 0.1$ the density profiles $\rho_{s}(r, t)$ and $\rho_{d}(r, t)$ cease to change with time and that the system becomes "arrested." One could interpret this state as the tagged particle remaining localized within the cage formed by the neighboring fluid particles. We discuss the significance of this phenomenon in Sec. VI. 

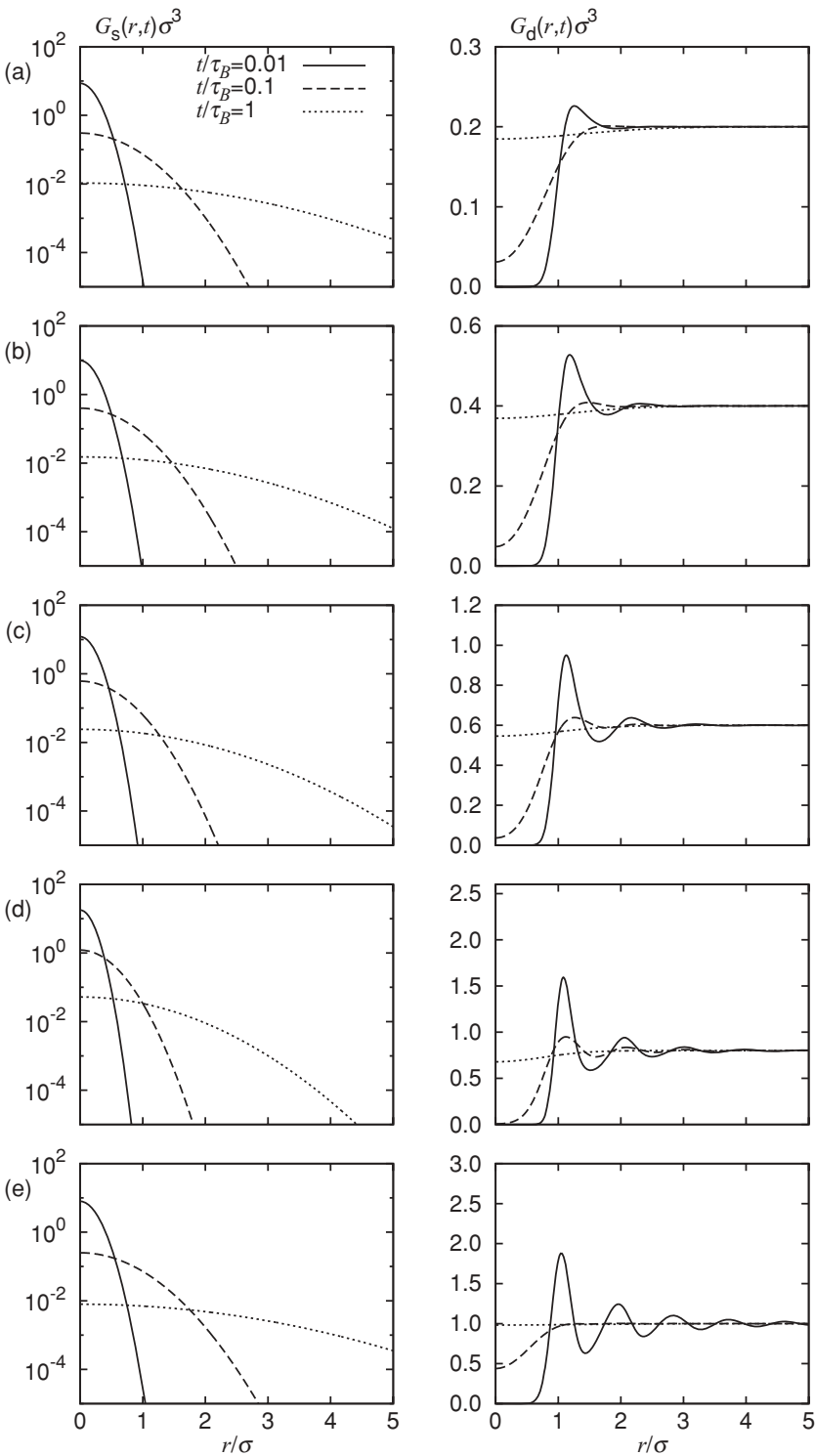

FIG. 4. The self and distinct parts of the van Hove distribution function, $G_{s}(r, t)$ and $G_{d}(r, t)$, calculated using the Vineyard approximation for densities: (a) $\rho \sigma^{3}=0.2$, (b) $\rho \sigma^{3}=0.4$, (c) $\rho \sigma^{3}=0.6$, (d) $\rho \sigma^{3}=0.8$, (e) $\rho \sigma^{3}=1$. The results are plotted for times $t / \tau_{B}=0.01$ (solid line), 0.1 (dashed line), and 1 (dotted line).

In Fig. 4 we show the van Hove functions calculated using the Vineyard approximation, Eqs. (17) and (19) with $D_{l}=D=k_{B} T \Gamma$, for fluid densities $\rho \sigma^{3}=0.2,0.4,0.6,0.8$, and 1 and times $t / \tau_{B}=0.01,0.1$, and 1 . As in the DDFT we have used $g(r)$ calculated using the RY functional and Percus' test particle method, although one could use $g(r)$ obtained from any reasonable method, including $g(r)$ measured in the BD simulations. Comparing the Vineyard results to the BD simulation results in Fig. 2, we find that there is reasonably good agreement between the two. The form of $G_{s}(r, t)$ is fixed to be Gaussian, so there is good agreement with $G_{s}(r, t)$ from the BD simulations, though it is clear that the width of $G_{s}(r, t)$ increases more rapidly in the Vineyard approximation. For densities $\rho \sigma^{3} \leq 0.8$ there is a similar amount of structure present in $G_{d}(r, t)$ for $r>\sigma$ in the Vineyard approximation as in the simulation results. However, for $\rho \sigma^{3}=1$ the

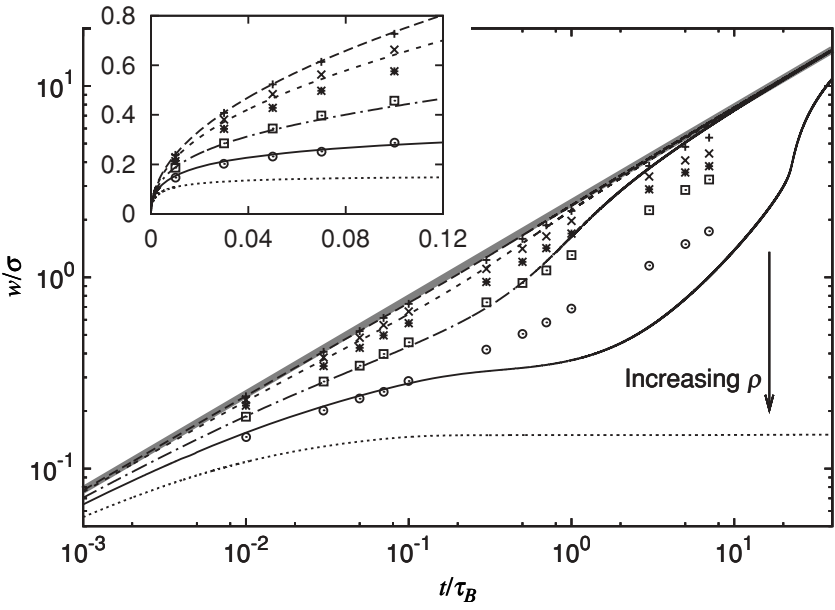

FIG. 5. The width $w$ of the self part of the van Hove function, defined in Eq. (11), measured in the BD simulations for $\rho \sigma^{3}=0.2(+), 0.4(\times), 0.6(*)$, $0.8(\square)$, and $1(\circ)$, and from the dynamical test particle theory, for $\rho \sigma^{3}=0.2$ (long dashed line), 0.4 (short dashed line), 0.6 (dashed-dotted line), 0.7 (solid line), and 0.8 (dotted line). Also shown is the result from the Vineyard approximation (thick solid grey line). In the main panel both axes are logarithmic; the inset displays the same results on linear scales.

Vineyard approximation does not exhibit the same degree of structure that is present in the simulation results.

In Fig. 5 we compare the width, $w(t)$, of the self part of the van Hove function, $G_{s}(r, t)$, obtained from (i) BD simulation results, (ii) dynamical test particle limit, and (iii) the Vineyard approximation. In the Vineyard approximation the time dependence of $w(t)$ is defined by Eq. (16) and does not depend on density, so there is only one master curve. This is because in the same way as in the dynamical test particle limit, we set $D_{l}=D$, where $D=k_{B} T \Gamma$ is the short time diffusion coefficient, which is strictly only equal to the long time selfdiffusion coefficient $D_{l}$ in the limit $\rho \rightarrow 0$. Since $w(t) \propto \sqrt{t}$, on the double logarithmic scale in Fig. 5 this is represented by a straight line with gradient $1 / 2$. We find that the simulation results are also approximately linear in this representation for all densities considered, but that there is a slowing down effect as density is increased, due to the fact that $D_{l}$ decreases as the fluid density is increased and is no longer equal to $D$. For $\rho \sigma^{3}=0.2$, the simulation results are close to the Vineyard result. As the bulk density is increased, the BD results move away from this line.

The dynamical test particle results for $w(t)$ in Fig. 5 exhibit a much stronger dependence on density. At low densities the curves are similar to the Vineyard and the BD results, but as the density is increased the $w(t)$ curves show a slowing down, and then (unphysical) speeding up of the dynamics, unlike that seen in the BD results. This slowing down is greatly exaggerated so that the DDFT curve for $\rho \sigma^{3}=0.6$ is similar to the BD result at $\rho \sigma^{3}=0.8$. Furthermore, the DDFT curves for $\rho \sigma^{3}=0.7$ and 0.8 have no counterpart in the simulation results. For $\rho \sigma^{3}=0.7$ the $w(t)$ curve shows extremely exaggerated slowing down and speeding up. We believe that the unphysical speeding up for $t / \tau_{B} \gtrsim 10$ is due to the fact that the DDFT incorrectly sets the long time diffusion coefficient $D_{l}$ equal to the short time diffusion 


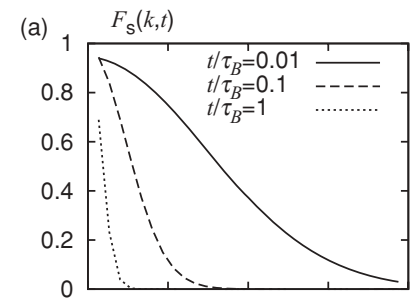

(b)

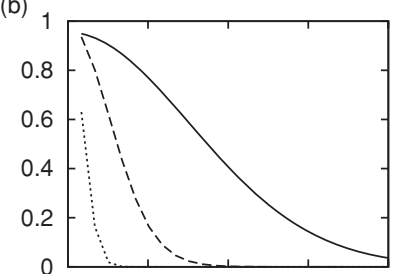

(c)

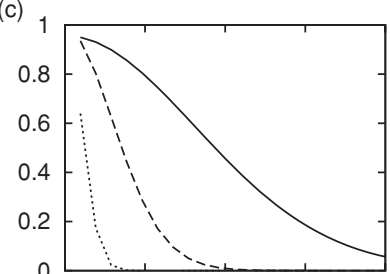

(d)

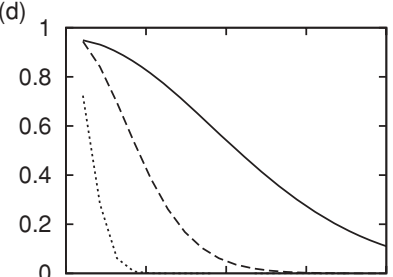

(e)

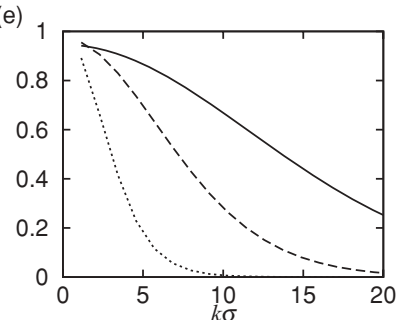

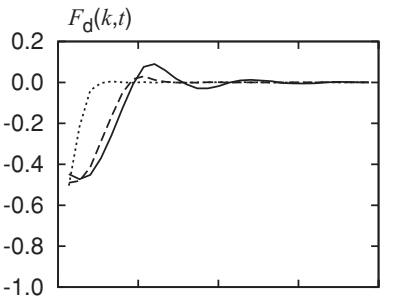
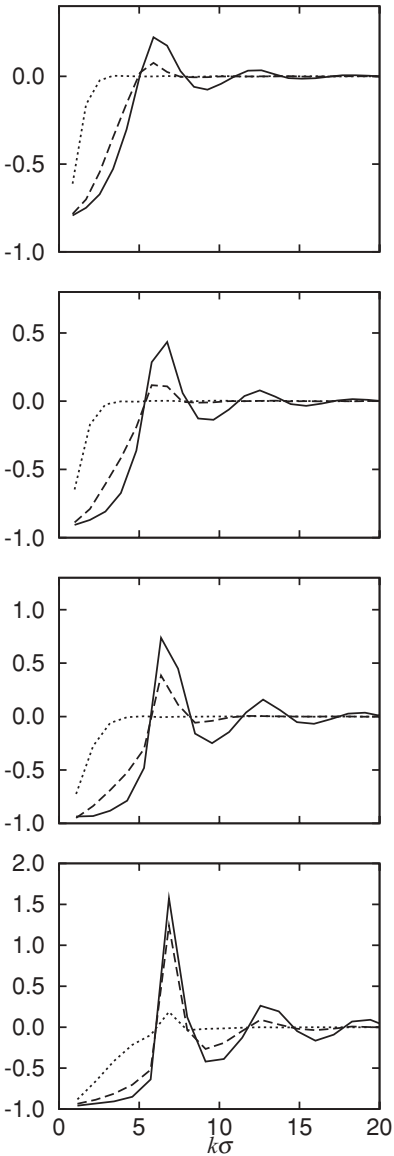

FIG. 6. Intermediate scattering function $F(k, t)$ as a function of the scaled wave vector $k \sigma$, obtained by a spatial Fourier transform, Eq. (12), of the BD simulation results for the van Hove functions displayed in Fig. 2.

coefficient $D$, so that as the particle escapes the cage of neighboring particles, it is forced to "catch-up" to give the incorrect long time behavior. Note that from the Smoluchowski Eq. (22) it can be shown ${ }^{51}$ that $w(t)$ must be sub-diffusive for intermediate times, and that the long time diffusion coefficient must be smaller than the short time one, a feature which is well established in the Brownian dynamics simulations and experiments. ${ }^{52-55}$ The curve for $\rho \sigma^{3}=0.8$ shows that the system slows down so much that the dynamics are arrested, so that $w(t \rightarrow \infty)$ is finite, as one would infer from the density profiles shown in Fig. 3. We postpone a discussion of the possible physical implications to Sec. VI.

For completeness, we also plot the intermediate scattering function $F(k, t)$. In Fig. 6 we display results from BD computer simulations, and in Fig. 7 the results from the DDFT. We find that for $\rho \sigma^{3}=0.2,0.4$, and 0.6 , the results from both approaches exhibit very similar structure. However, since in the DDFT the dynamics become arrested at
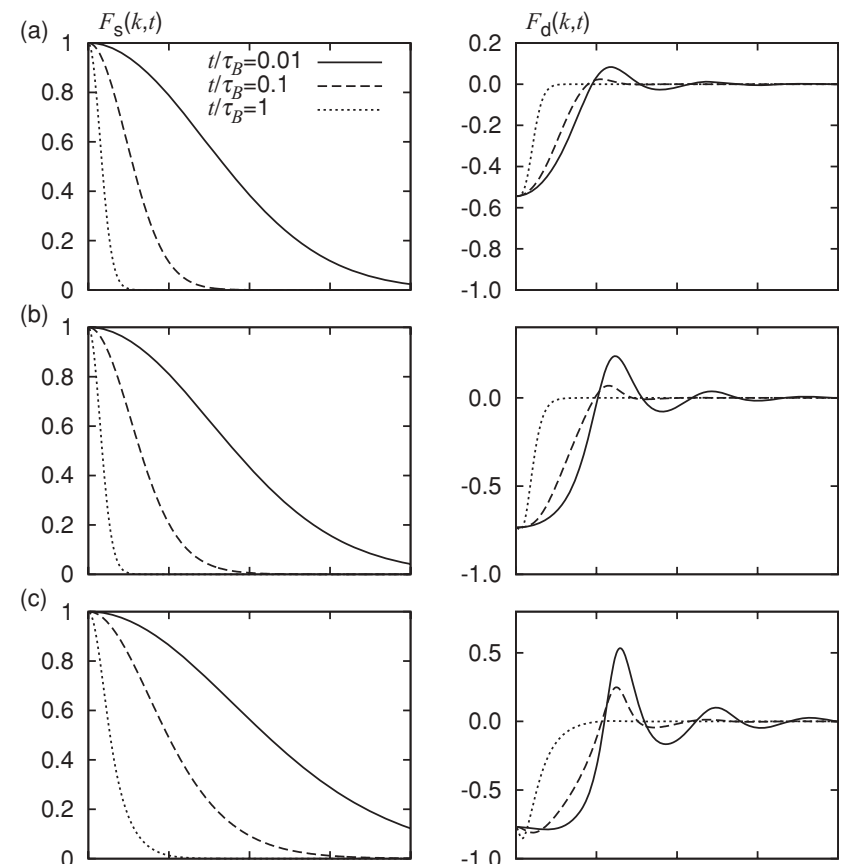

(d)
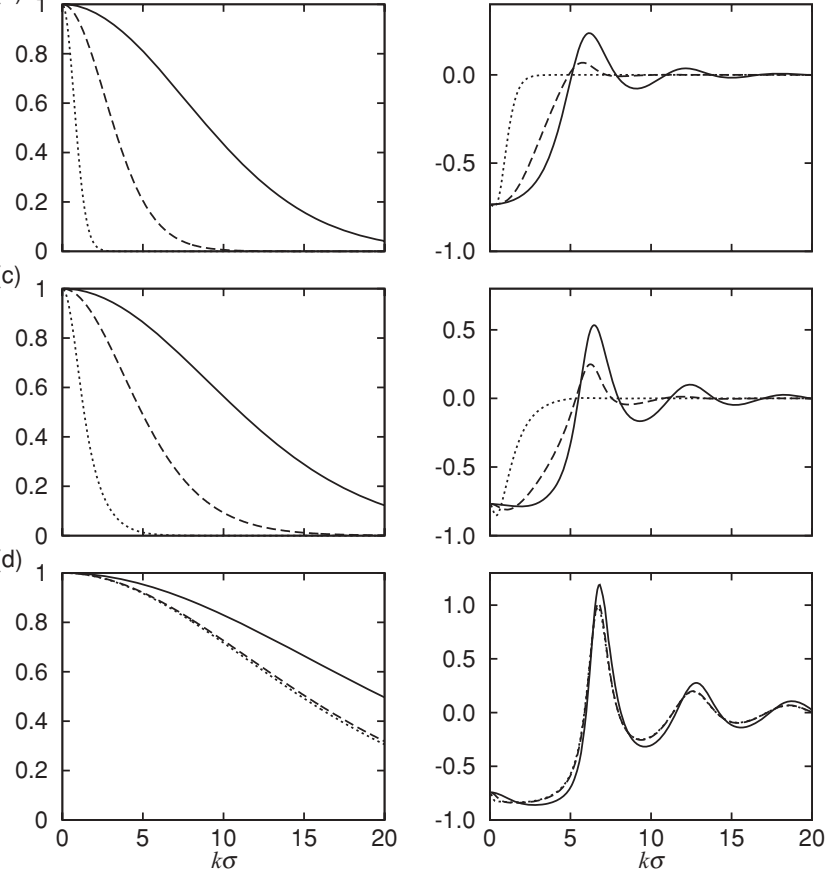

FIG. 7. Intermediate scattering functions $F(k, t)$, obtained by a spatial Fourier transform, Eq. (12), of the dynamic test particle density profiles, $\rho_{s}(r, t)$ and $\rho_{d}(r, t)$, displayed in Fig. 3.

$\rho \sigma^{3}=0.8$, so $F(k, t)$ becomes arrested after a very short time, unlike the BD simulations result. In Fig. 8 we plot the scaled intermediate scattering function,

$$
\phi(k, t)=\frac{F_{s}(k, t)}{F_{s}(k, t=0)},
$$

for fixed $k \sigma=2 \pi$, obtained from the Vineyard approximation and compare to the BD simulation results [Fig. 8(a)] and the DDFT results [Fig. 8(b)]. At the lower densities the BD simulation results and the DDFT results are close to the Vineyard approximation and both show some slowing down with density. At the higher densities the BD results continue to show a steady decay. However, in the DDFT results for $\rho \sigma^{3}=0.7$ we see $\phi(k \sigma=2 \pi, t)$ decays in two stages over a much longer time. For $\rho \sigma^{3}=0.8$ the arrested dynamics cause $\phi(k \sigma=2 \pi, t)$ to remain finite in the limit $t \rightarrow \infty$.

\section{B. Relating dynamic to static density profiles}

One may connect DDFT and equilibrium DFT by finding the unique set of effective external potentials $\left\{u_{i}(\mathbf{r})\right\}$ that in equilibrium generate the same set of density profiles as obtained in the dynamic approach at a particular time $t$. These potentials represent the net effect of finite time and limited diffusion preventing the fluid from finding the structure that 

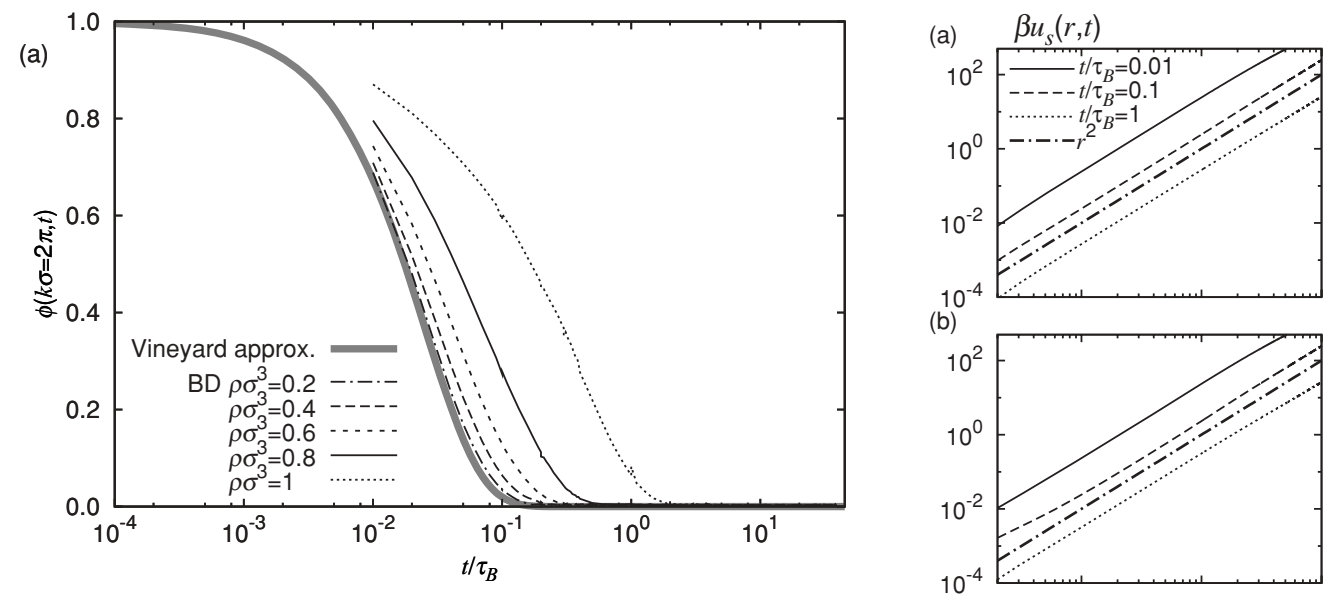

(c)
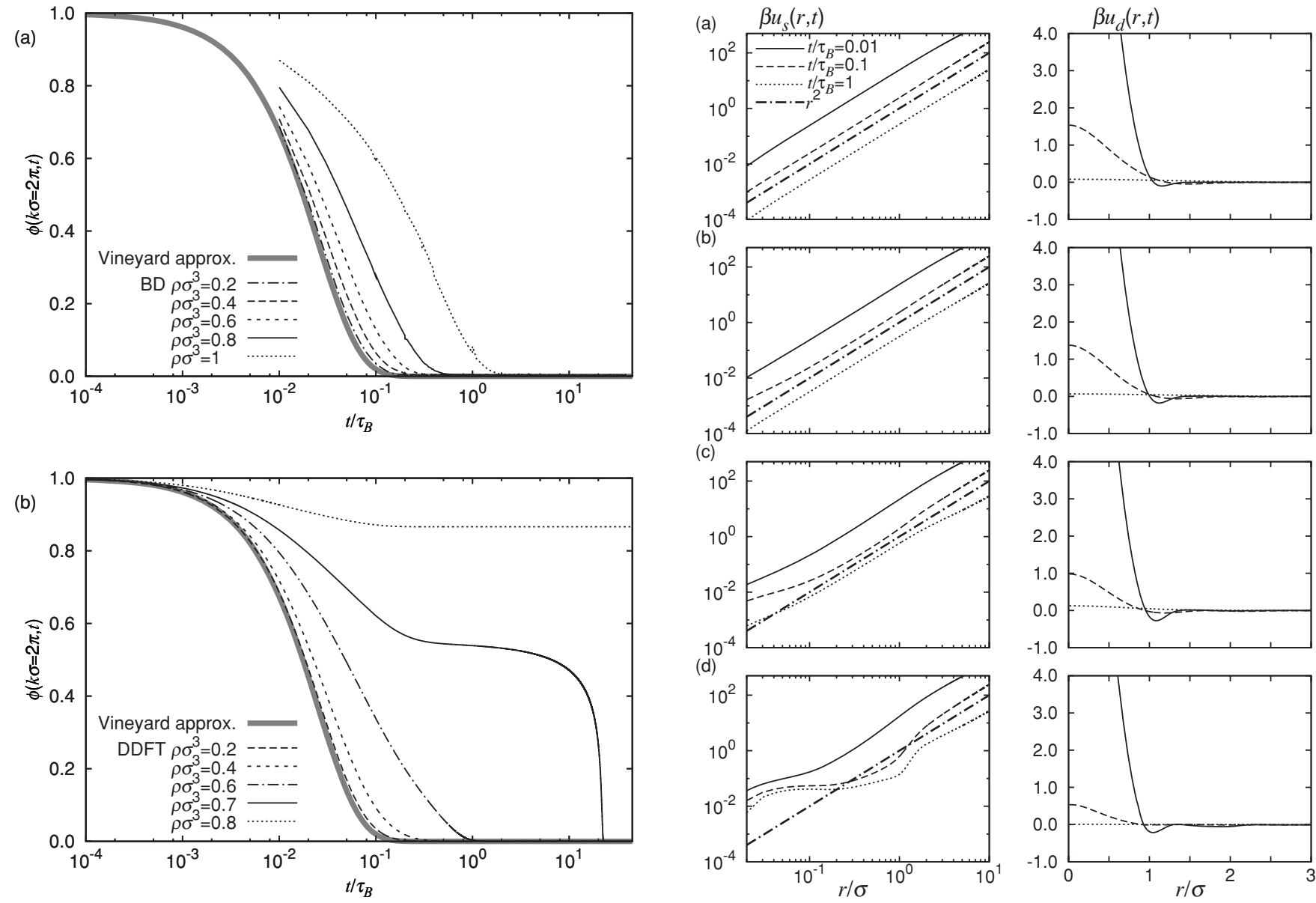

FIG. 8. Scaled intermediate scattering function $\phi(k \sigma=2 \pi, t)$ as a function of time $t / \tau_{B}$, calculated from the Vineyard approximation (thick gray line) compared to (a) BD simulation results and (b) the dynamical test particle method. The dynamical test particle results exhibit slowing and arrested dynamics for $\rho \sigma^{3}=0.7$ and 0.8 .

minimizes the system free energy. For the two-component system considered here, the two external potentials $\beta u_{i}(r, t)$ may be recovered, up to an overall time-dependent additive constant $\beta \mu_{i}(r, t)$, by rearranging Eq. (33)

$$
\beta u_{i}(r, t)-\beta \mu_{i}=c_{i}^{(1)}\left(r ;\left[\rho_{s}, \rho_{d}\right]\right)-\ln \left[\Lambda^{3} \rho_{i}(r, t)\right],
$$

where $\rho_{s}(r, t)$ and $\rho_{d}(r, t)$ are the solution of the DDFT at time $t$. In Fig. 9 we plot these external potentials corresponding to the density profiles from DDFT displayed in Fig. 3. We find that at the lowest densities $\rho \sigma^{3}=0.2$ and 0.4 , the shape of $u_{s}(r, t)$ is approximately parabolic for all times and distances $r$. As the fluid density is increased, $u_{s}(r, t)$ departs from the parabolic form. For $\rho \sigma^{3}=0.8$ the curves are still parabolic at large $r$, but at small $r$ they become distorted. We find that $u_{d}(r, t)$ does not vary significantly with density. At short times it is dominated by strong repulsion within the hard-core diameter, $r<\sigma$. Recall that in order to calculate $g(r)$, which corresponds to $t=0$, we chose to use Percus' test particle method, and hence have introduced an external potential equal to the hard sphere potential. The strong repulsion found for short times is a remnant of this external potential. As $t$ increases, the strength of this repulsion decreases and becomes almost zero for $t / \tau_{B}=1 .^{56}$

FIG. 9. External potentials, $u_{s}(r, t)$ and $u_{d}(r, t)$, required to generate equilibrium density profiles, $\rho_{s}(r, t)$ and $\rho_{d}(r, t)$, identical to those obtained from the dynamical test particle approach - see Fig. 3. The external potentials also include an overall time-dependent additive constant which is not indicated. Note the logarithmic $x$-axis for $u_{s}(r, t)$. At low densities $u_{s}(r, t)$ is approximately parabolic, as indicated by the straight line (dashed-dotted). At high densities $u_{s}(r, t)$ is distorted at small $r$, but still parabolic at large $r$.

\section{Corresponding equilibrium approach}

Having established the form of the external potentials necessary to create equilibrium fluid density profiles equal to the profiles calculated using the dynamical test particle method, we now seek a simple approximation for this set of external potentials, to allow us to easily calculate equilibrium density profiles that mimic the dynamic profiles. In other words, we seek to determine the full van Hove function when $G_{s}(r, t)$ has a given width $w$, without calculating the entire preceding time series of profiles. In doing this we lose time $t$ as a function argument and instead we must "label" the density profiles with $w$. In what follows, we will disregard the associated problem of relating $w$ to time $t$.

We parametrize the external potentials using a simple functional form. First, we assume that $u_{s}(r, w)$ is parabolic for all widths

$$
\beta u_{s}(r, \lambda)=\lambda r^{2},
$$

where $\lambda$ is the strength of the confining potential, and $w$ is now an unknown function of $\lambda$. For the external potential that acts on species $d$ we consider two options. The first is to 
assume that $u_{d}(r, w)=0$. In this case, it is possible to solve exactly for the equilibrium distribution functions and the free energy, as outlined in Appendix A. We find that the species $s$ density profile is a Gaussian,

$$
\rho_{s}(r, \lambda)=\frac{\exp \left(-\beta \lambda r^{2}\right)}{(\pi / \beta \lambda)^{3 / 2}},
$$

where the dependence of the width $w$ on $\lambda$ is

$$
w(\lambda)=(2 \lambda / 3)^{-1 / 2},
$$

and the $d$ profile is given by a convolution of the radial distribution function $g(r)$, together with the Gaussian profile $\rho_{s}(r)$, and multiplied by $\rho$

$$
\rho_{d}(r)=\rho \int \mathrm{d} \mathbf{r}^{\prime} \rho_{s}\left(\mathbf{r}^{\prime}\right) g\left(\left|\mathbf{r}-\mathbf{r}^{\prime}\right|\right)
$$

These distribution functions are identical to those from the (dynamic) Vineyard approach.

The second approach that we consider is to calculate the density profiles without defining the external potential $u_{d}(r, w)$ at the outset of the calculation. Instead, we determine this potential self-consistently "on-the-fly" as part of our iterative numerical solution routine, based on the following considerations: First, recall the normalization constraints on the van Hove function in Eqs. (7) and (8). In order to satisfy the normalization constraint (7) on the density profile for the single tagged $s$ particle, we introduce a Lagrange multiplier $\mu_{s}$. One may also consider $\lambda$ to be a Lagrange multiplier that enforces the width constraint (11) on the profile $\rho_{s}(r)$. In our calculations the value of $\mu_{s}$ is determined on-the-fly by enforcing Eq. (7) at each step of our iterative routine. However, we are not able to do the same for the density profile of the remaining $d$ particles, because we also must have

$$
\rho_{d}(r, w) \rightarrow \rho, \text { as } r \rightarrow \infty
$$

Multiplying $\rho_{d}(r)$ by a single factor breaks this condition, so we are not able to simply enforce (8) at each step of our iterative routine in the same way as we do for $\rho_{s}(r)$. The condition in Eq. (64) implies that we require an a priori unknown inhomogeneous external potential, $u_{d}(r, w)$, with the property that $u_{d}(r, w) \rightarrow 0$ as $r \rightarrow \infty$. This may be achieved by scaling the quantity $\rho_{d}(r)-\rho$ (instead of scaling $\rho_{d}(r)$ itself) at each step, so that $\rho_{d}(r)$ satisfies both Eqs. (8) and (64). Once convergence of the numerical procedure is achieved, one may then inspect the effective external potential $u_{d}(r, w)$ by substituting the resulting density profiles into Eq. (59).

The density profiles calculated using this equilibrium method are shown in Fig. 10 where we plot $\rho_{d}(r, w)$ and $\rho_{s}(r, w)$ having widths identical to those of the van Hove functions from simulation, displayed in Fig. 2. Note that we consider only the densities, $\rho \sigma^{3}=0.2,0.4,0.6$, and 0.8 . These equilibrium profiles have been calculated using a normalization constant taken from the approximation for $g(r)$ calculated using Percus' test particle method. We find that there is reasonable qualitative agreement between the equilibrium DFT density profiles displayed in Fig. 10 and the BD simulation results displayed in Fig. 2. However, the profiles predict too much infilling in the region close to the origin $r$ $<\sigma$, particularly at higher densities, which in turn results in
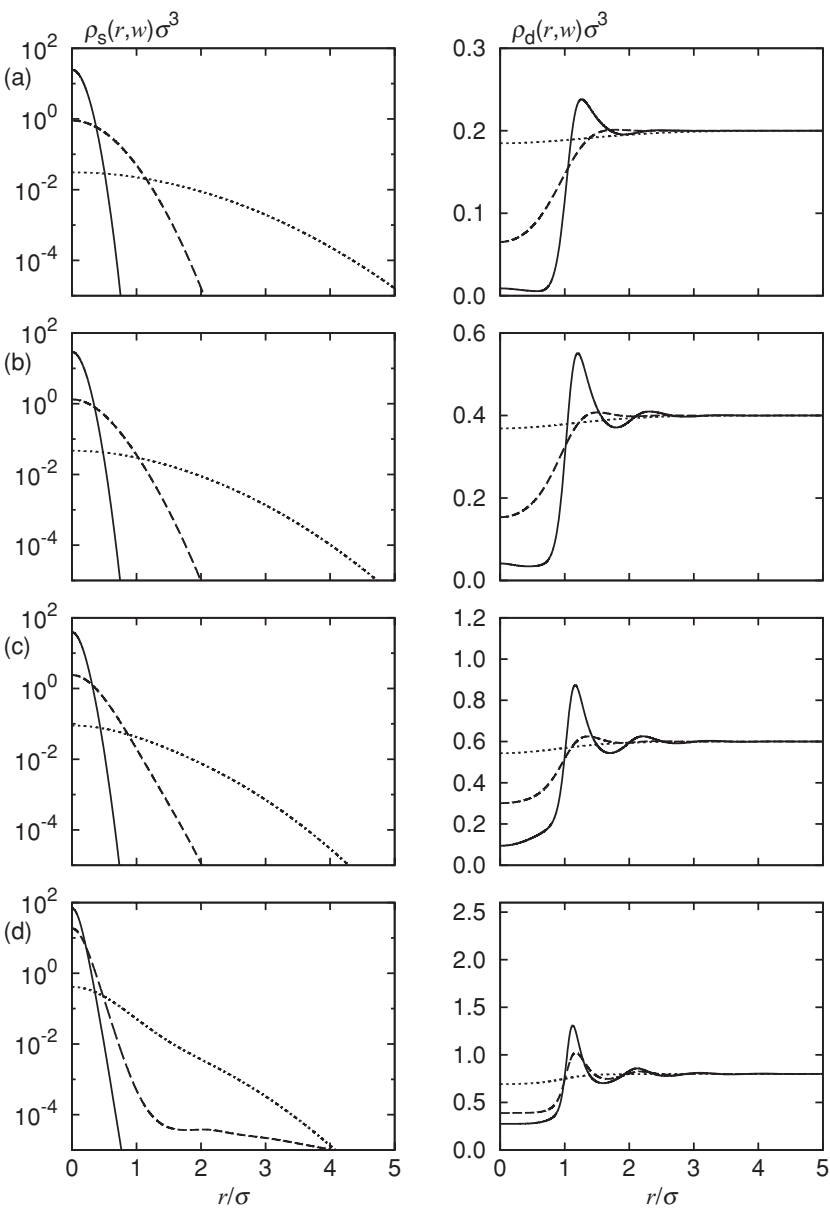

FIG. 10. The density profiles $\rho_{s}(r, w)$ and $\rho_{d}(r, w)$, calculated using the equilibrium DFT. The curves are calculated at the densities: (a) $\rho \sigma^{3}=0.2$, (b) $\rho \sigma^{3}=0.4$, (c) $\rho \sigma^{3}=0.6$, and (d) $\rho \sigma^{3}=0.8$. The curves are chosen so that the widths $w$ of $\rho_{s}(r, w)$ correspond to the same widths of $G_{s}(r, t)$ at times $t / \tau_{B}=0.01$ (solid line), 0.1 (dashed line), and 1 (dotted line) displayed in Fig. 2.

an underestimate in the structure of the profiles at larger $r$ $>\sigma$. This error occurs for the reasons discussed in Sec. IV C; i.e., the RY functional does not exert a strong enough interaction from the test particle onto the rest of the fluid.

For $\rho \sigma^{3}=0.8$, shown in Fig. 10(d), we are able to calculate density profiles for all values of $w$, even though in the DDFT the profiles became "trapped" at small $w$. The most striking aspect of these density profiles is that for intermediate values of $w$ we see that $\rho_{s}(r)$ exhibits a plateau and a long tail. These features were not observed in the BD simulation results. However, similar features are present in $G_{s}(r, t)$ at intermediate times for colloidal spheres at densities close to the glass transition, where they are interpreted as the signature of dynamical heterogeneity in the system. ${ }^{3}$

\section{Free energy landscape}

Since we are able to convert the dynamic density profiles into their equilibrium equivalents via a set of external potentials, cf. Eq. (59), we are also able to calculate the equilibrium Helmholtz free energy for this corresponding equilibrium situation. Although this free energy is strictly an equilibrium 


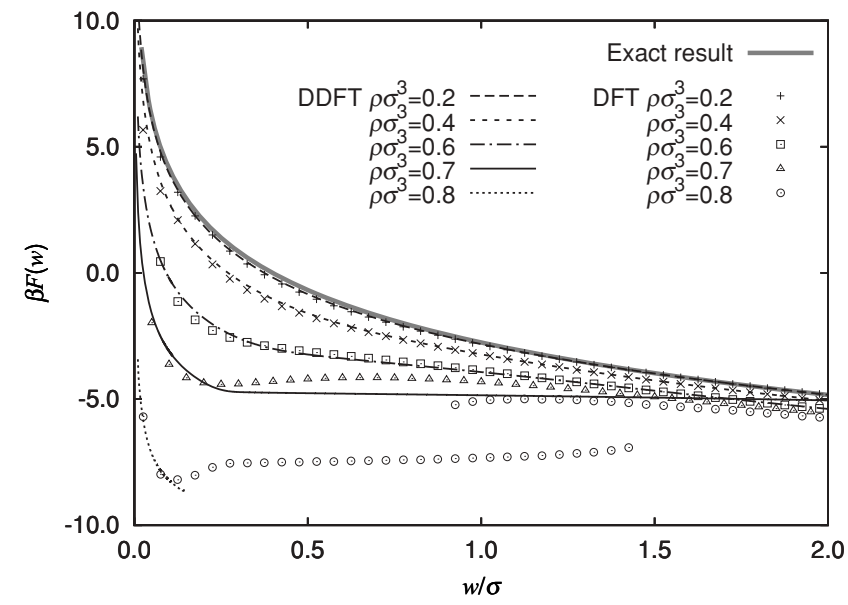

FIG. 11. The free energy landscape $F(w)$ plotted as a function of $w$, the width of $\rho_{s}(r, w)$, calculated using both the DDFT and equilibrium DFT approaches, compared to the exact result, Eq. (65). For $\rho \sigma^{3}=0.8$ we find two disconnected branches of $F(w)$.

quantity, since it underlies the time evolution of our dynamic approach we believe that it plays a relevant role. Therefore, by substituting the density profiles calculated using the DDFT into the free energy functional, Eqs. (30) and (53), we are able to map out a "free energy landscape" as a function of $t$ or $w$. Figure 11 plots this free energy landscape, $F(w)$, for densities $\rho \sigma^{3}=0.2,0.4,0.6,0.7$, and 0.8 . For $\rho \sigma^{3}=0.2,0.4$, and 0.6 we find that $F(w)$ decreases monotonically with $w$. This decrease is initially steep and then the gradient begins to reduce as $w$ increases. For $\rho \sigma^{3}=0.7$, we find that after the initial steep descent the landscape develops an almost constant plateau, but there is still a very small negative gradient. For $\rho \sigma^{3}=0.8$ the decrease is rapid and then the landscape terminates abruptly as the density profiles reach an arrested state.

In the equilibrium case where $u_{d}(r, w)=0$, one has an exact solution (see Appendix A) for the free energy landscape as a function of the width,

$$
F(w)=F_{\text {id }}-\ln \left(Z_{N}^{\prime}\right)-\frac{3}{2} \ln \left(\frac{2 \pi w^{2}}{3 \beta}\right),
$$

where $F_{\text {id }}$ is the ideal Helmholtz free energy, and $Z_{N}^{\prime}$ is an irrelevant constant representing the partition function of the fluid when the test particle is located at the origin. We plot Eq. (65) alongside the landscapes from the DDFT approach in Fig. 11. We find that this curve is located close to the DDFT landscape for $\rho \sigma^{3}=0.2$ but that the deviation grows with increasing density.

We also calculate the free energy landscape via the equilibrium approach described in the previous subsection and compare this to the results from both the dynamic approach and the exact equilibrium result in Fig. 11. For the lower densities, $\rho \sigma^{3}=0.2,0.4$, and 0.6 , we find that there is good agreement between the DDFT and equilibrium DFT approaches. For $\rho \sigma^{3}=0.7$ we find that there is good agreement at low $w$, but around $w / \sigma=0.7$ there is a local maximum in $F(w)$ in the equilibrium DFT results that is not present in the DDFT results. For $\rho \sigma^{3}=0.8$ we find that the DDFT free energy landscape terminates abruptly at a fairly low value of $w$.

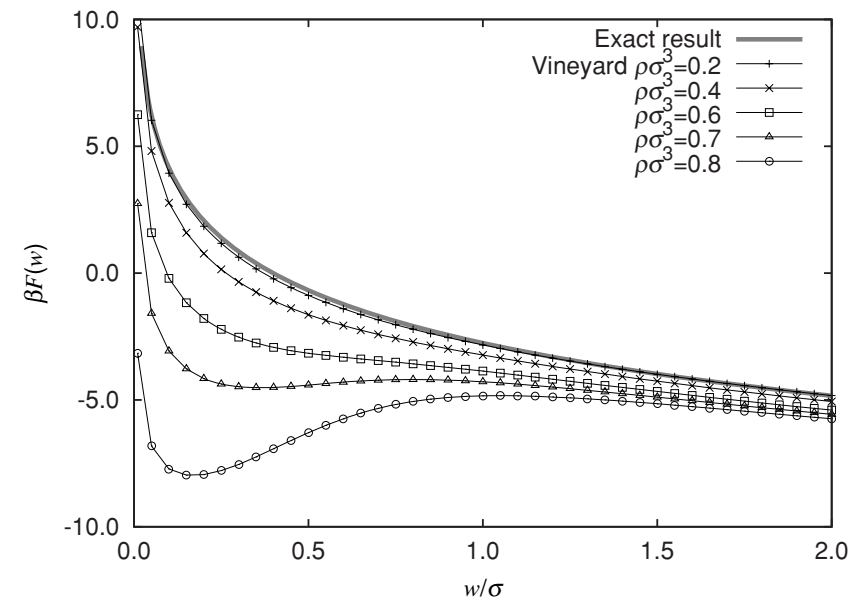

FIG. 12. The free energy landscape $F(w)$ plotted as a function of the width $w / \sigma$, calculated by substituting the density profiles from the exact equilibrium solution [Eqs. (61) and (63)] into the RY functional. Here the deviation from the exact free energy and the emergence of the minimum are entirely due to the RY functional.

However, since we can calculate the density profiles using our equilibrium approach for all widths we can therefore calculate $F(w)$ for all $w$. We find a free energy landscape with two disconnected branches. Therefore, for a range of values of $\lambda$ we find two solutions to the Euler-Lagrange equations with different widths. Whether this is an indication of dynamic heterogeneity or an artefact of the functional is an interesting question.

If we take the density profiles calculated using the exact route [Eqs. (61) and (63)] and substitute these into the RY functional, we find that the free energy curves, shown in Fig. 12, do not follow the exact result (65), but are very similar to those from the DDFT and DFT approaches and even exhibit minima for $\rho \sigma^{3}=0.7$ and 0.8 . Therefore, we must conclude that it is largely a property of the RY approximation for the free energy functional that generates these minima.

In Fig. 13 we plot the exact relationship, Eq. (62), between the strength of the confining potential, $\lambda$, against the width, $w$, and compare it to the results from the equilibrium DFT approach. At the lowest densities the equilibrium DFT approach closely follows the exact result, but as the density is increased the width decreases for a given $\lambda$. For $\rho \sigma^{3}=0.8$ we find that this curve is no longer monotonic and has two disconnected branches.

Systems which are in a glassy or jammed state are by definition nonergodic. A way of modeling non ergodicity in Brownian dynamics is to measure the state of the system over too short a time frame. We demonstrate this effect by simulating a fluid where a single tagged particle is trapped in a parabolic potential well; cf. Eq. (60). If the radius of the well is sufficiently large and the simulation time is too small, then the particle is not able to fully explore the outer regions of the potential well. In the inset of Fig. 13 we plot two results pertaining to this scenario where the fluid density is $\rho \sigma^{3}=0.8$, and $t^{*}$ is the simulation run time. The results for the longer simulation run time agree well with the exact result, but results over the shorter time underestimate the width, particularly at the smaller values of $\lambda$. A similar effect may exist in 


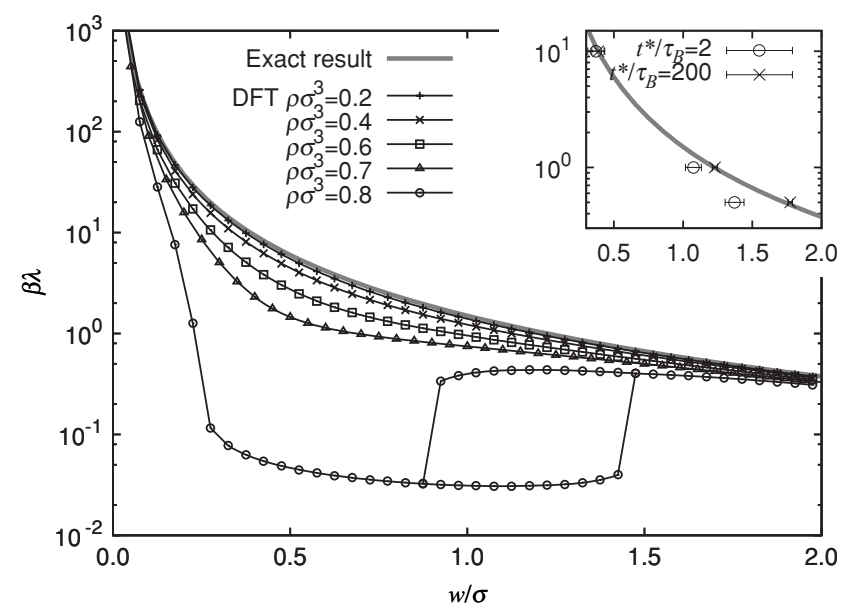

FIG. 13. The (scaled) strength of the parabolic potential, $\beta \lambda$, versus the resulting width, $w / \sigma$, of $\rho_{s}(r)$. Results are calculated using the equilibrium DFT and compared to the exact result. For the bulk fluid densities $\rho \sigma^{3}=0.2$, $0.4,0.6$, and $0.7, \lambda$ decreases monotonically with $w$. However, for $\rho \sigma^{3}=0.8$ the curve is no longer monotonic, and exhibits two disconnected branches. The inset shows the effect of insufficient simulation run times on the equivalent situation studied using BD computer simulations, where $t^{*}$ is the simulation run time.

the DFT approach, where the non ergodicity arises from not including the states where the particle is far from the origin. Recall that formally the density profile from DFT is an average over all possible states of the system. Using an approximate functional some of these states may be neglected. ${ }^{57}$

\section{CONCLUDING REMARKS}

On the basis of the theoretical and simulation results that we present in this paper for the dynamics of the bulk hard sphere fluid, we conclude that the dynamical test particle limit, combined with DDFT, provides a reliable method for calculating the van Hove (and other related) dynamical pair correlation functions at low and at intermediate densities $\rho \sigma^{3} \lesssim 0.6$. In the previous publication ${ }^{17}$ we have shown that the theory may be applied in a fairly straightforward manner also to inhomogeneous situations, hence we conclude that the dynamic test particle theory may indeed be used to calculate the van Hove function for fluids at interfaces and under confinement. Furthermore we have shown that, quite surprisingly, at low and intermediate densities the very simple Vineyard approximation (reviewed in Sec. II C) is actually quantitatively fairly good. This approximation only requires as input the radial distribution function $g(r)$ and the diffusivity $D_{l}$ and therefore provides a very useful quick approach for obtaining an approximation for the van Hove function for colloidal fluids.

The possible conclusions about the performance and even about the qualitative status of the predictions of the theory at higher densities are far more intricate though. In this regime, the theory in its current form is clearly quantitatively unreliable-compare for example, the results from BD simulations in Fig. 2(d) to those from the dynamic test particle theory shown in Fig. 3(d) for $\rho \sigma^{3}=0.8$, where the theory predicts that the system jams, whereas in simulations the sys- tem is an ergodic fluid. Moreover, at even higher densities, the monodisperse hard sphere system crystallizes in simulations rather than undergoing a glass transition; polydispersity would be required to suppress freezing. ${ }^{58}$ We believe that the behavior of the theory at higher densities can primarily be ascribed to our choice of approximation for the free energy functional-see Sec. IV B for the reasons for using the RY functional (53) in the present study. We believe that if we had used a more accurate functional, such as Rosenfeld's fundamental measure theory, ${ }^{59-61}$ quantitatively more accurate results could be obtained at these higher densities. Nevertheless, the theory yields a clear prediction that there is a dynamic (glass) transition, whereby the tagged (self) particle becomes trapped in the cage formed by the surrounding particles. We can offer three possible interpretations of this result.

First, one may conclude that this glass transition stems simply from the use of the approximate RY functional and since the theory predicts the glass transition to be at a density value that is well below where the glass transition is believed to occur, our results at higher densities should be disregarded.

An alternative conclusion that one may draw is that the theory is correctly describing (some of) the physics of the glass transition, but that the predictions are only qualitative in nature and occur in reality at higher densities and in polydisperse systems. Support for this point of view comes in particular from results such as those in Fig. 8, where we display results for $\phi(k \sigma=2 \pi, t)$. For $\rho \sigma^{3}=0.7$, which is near to where the theory predicts the glass transition to be, we find a plateau in $\phi(k, t)$ and the clear presence of two-stage relaxation, which indeed has been observed in hard sphere colloidal suspensions. ${ }^{9}$ Hence our results are (qualitatively) similar to those from MCT. ${ }^{6,9}$ Further support for the above interpretation comes from the results in Figs. 11 and 12 for the behavior of the free energy landscape that underlies the DDFT. The appearance of a minimum in the free energy as a function of displacement, corresponding to a particle becoming trapped in the cage formed by its neighbors, is one central prediction of the theory by Schweizer and Saltzmann, ${ }^{62,63}$ who combined elements of MCT, DFT, and activated rate theory, in order to describe localization and transport in glassy fluids. Furthermore, given some of the work in the literature based on DFT to study the glass transition, our prediction that particles become localized should not come as a surprise: Wolynes and coworkers ${ }^{64-66}$ developed a successful model of hard sphere vitrification, which is similar to the DFT treatment of crystallization. ${ }^{1,24}$ Using a random closepacked, non periodic lattice they found a fluid-glass transition where the fluid "crystallizes" onto this lattice. The success of this method, along with its ability to model the freezing transition (onto a regular lattice), has provoked a number of further developments. ${ }^{67-71}$ Other approaches ${ }^{72}$ have investigated dense Brownian systems through modeling via certain stochastic differential equations and found that the system exhibits glassy behavior. Thus, overall our results seem to be qualitatively consistent with other DFT based theories and with MCT for the glass transition. Nevertheless, the density where the glass transition occurs, as predicted by the theory in its present form, is far too low. Furthermore, it could be the case that the similarity between our results and those from 
the MCT are somehow a mathematical (rather than physical) coincidence, since an essential feature of MCT is the presence of memory in the dynamical equations. This important feature is absent from the present DDFT.

The third possible conclusion that one may draw concerns is the question whether the minimum in the free energy and the localization of the tagged self particle are merely a signature of freezing in the theory. The RY functional is well known to predict the freezing transition to occur at a density below that where it occurs in reality. It could simply be the case that this functional overly favors freezing, so that when it is applied in the way we use it here, where we constrain all density profiles, $\rho_{s}(r)$ and $\rho_{d}(r)$, to be spherically symmetric, a signature of freezing shows up as the tagged self particle becoming localized.

Some merit can be found in all of the arguments outlined above and we find ourselves unable to judge which one(s) are correct. Indeed further work is required to provide a clear assessment of these issues. In particular, the dynamical test particle theory should be implemented with a more sophisticated approximation for the free energy functional than we have used here.

As we have shown, our approach is based on integrating the Smoluchowski equation (22) over all except one of the position coordinates, in order to derive an equation for the one-body density distribution. An alternative approach is to integrate over all but two of the position coordinates, in order to obtain [c.f. Eq. (23)] an equation for the two-body distribution function $\rho^{(2)}\left(\mathbf{r}_{1}, \mathbf{r}_{2}, t\right)$ $=N(N-1) \iint \mathrm{d} \mathbf{r}_{3} \ldots \int \mathrm{d} \mathbf{r}_{N} P\left(\mathbf{r}^{N}, t\right)$. The resulting dynamical equation depends on the three-body distribution function $\rho^{(3)}\left(\mathbf{r}_{1}, \mathbf{r}_{2}, \mathbf{r}_{3}, t\right)$. On making a suitable closure approximation, this provides a different starting point for studying the pair correlations in a colloidal fluid-see e.g. Refs. 52 and 53 and references therein, which also consider the effect of the hydrodynamic interactions between the colloids. Developing the theory for the dynamical pair correlation functions in this way is very natural. However, we believe that the strength of our method, where we use the dynamical test particle approach allowing us to work at the one-body level, is that we are able to use DFT to close our equations and therefore we are able to describe the fluid spatial correlations very accurately.

Finally, we mention other possible directions for developing the theory in the future. One important aspect in the dynamics of colloidal dispersions, that we have entirely neglected here, are the hydrodynamic interactions between the particles. Rex and Löwen ${ }^{73,74}$ have shown how to include the hydrodynamic interactions in a DDFT treatment and so it would be worthwhile to use their DDFT formulation together with the present dynamical test particle limit, in order to calculate the van Hove function under the influence of hydrodynamic interactions.

A further aspect of our work that offers possible extensions of the theory, concerns the question how to model the diffusivity of the tagged particle in a better way. In the dynamical test particle calculation one could replace the (constant) diffusion coefficient in Eq. (48) with a diffusion coefficient that depends on time; i.e., to replace $D \rightarrow D(t)$. In doing this one could ensure that $D(t)$ takes the correct values at both short and long times. However, doing this still does not treat memory effects in the dynamics. As MCT demonstrates, memory effects are key for a system to exhibit the ideal glass transition scenario. ${ }^{9,51,75}$ Thus, we believe that including memory into our theory would be a crucial step in future work. This could possibly be done along the lines of the interesting work of Medina-Noyola and coworkers. ${ }^{12-16}$ To include memory in our theory one could replace Eq. (29) with $^{16,76,77}$

$$
\begin{aligned}
\frac{\partial \rho_{i}(\mathbf{r}, t)}{\partial t}= & \nabla \cdot \int_{0}^{t} d t^{\prime} \int \mathrm{d} \mathbf{r}^{\prime} \Gamma\left(\mathbf{r}-\mathbf{r}^{\prime}, t-t^{\prime}\right) \\
& \times\left[\rho_{i}\left(\mathbf{r}^{\prime}, t^{\prime}\right) \nabla \frac{\delta F\left[\left\{\rho_{i}\right\}\right]}{\delta \rho_{i}\left(\mathbf{r}^{\prime}, t^{\prime}\right)}\right],
\end{aligned}
$$

where the mobility coefficient $\Gamma$ has been replaced by one that is non local in time and space. However, this would result in a considerable increase in computational complexity as within DFT the correlations in space are already treated in a complex manner and these would need to be coupled to the correlations in time. Whether such non locality helps to cure some of the deficiencies of our approach is an open question.

\section{ACKNOWLEDGMENTS}

P.H. thanks the EPSRC for funding under Grant EP/E065619/1 and A.J.A. gratefully acknowledges financial support from RCUK M.S. and A.F. thank the DFG for support via SFB840/A3.

\section{APPENDIX A: EXACT RESULTS}

We consider a fluid of $N$ particles with positions $\mathbf{r}_{i}$, momenta $\mathbf{p}_{i}$, and mass $m$ in the presence of an arbitrary external field that acts only on particle $i=1, u_{1}(\mathbf{r})=\lambda \mathbf{r}_{1}^{2}$. Assuming that we are in the classical limit, the Hamiltonian is given by $H_{N}=K+V+U$ where the contributions are due to the (classical) kinetic energy, the total inter particle potential (not necessarily pairwise additive), and the external potential, respectively;

$$
\begin{aligned}
K & =\sum_{i=1}^{N} \frac{\mathbf{p}_{i}^{2}}{2 m} \\
V & =v\left(\mathbf{r}_{1}, \ldots, \mathbf{r}_{N}\right) \\
U & =\lambda \mathbf{r}_{1}^{2} .
\end{aligned}
$$

The canonical partition function, $Q_{N}(V, T)$, is given by

$$
Q_{N}(V, T)=\frac{h^{-3 N}}{(N-1) !} \iint \mathrm{d} \mathbf{r}^{N} \mathrm{~d} \mathbf{p}^{N} \exp \left[-\beta H_{N}\left(\mathbf{r}^{N}, \mathbf{p}^{N}\right)\right]
$$

where $h$ is Planck's constant, and the $(N-1)$ ! factor results from the fact that besides particle $i=1$, the remaining particles are indistinguishable. The integrations over momenta in Eq. (A4) can be carried out explicitly, leaving a configuration 
integral over positional degrees of freedom:

$$
Z_{N}=\int \mathrm{d} \mathbf{r}_{1} \ldots \mathrm{d} \mathbf{r}_{N} \exp (-\beta(V+U)) .
$$

Note that for Brownian particles $Z_{N}$ is also the quantity that characterizes the structure of the fluid. Substituting our external potential (A3) into (A5) we obtain

$$
\begin{aligned}
Z_{N} & =\int \mathrm{d} \mathbf{r}_{1} \exp \left(-\beta \lambda \mathbf{r}_{1}^{2}\right) \int \mathrm{d} \mathbf{r}_{2 \ldots N} \exp \left(-\beta V\left(\mathbf{r}^{N}\right)\right) \\
& =\int \mathrm{d} \mathbf{r}_{1} \exp \left(-\beta \lambda \mathbf{r}_{1}^{2}\right) \int \mathrm{d} \mathbf{r}_{2 \ldots N}^{\prime} \exp \left(-\beta V\left(\mathbf{r}_{2 \ldots N}^{\prime}\right)\right),
\end{aligned}
$$

where in the second step we have made the substitution $\mathbf{r}_{i}^{\prime}$ $=\mathbf{r}_{i}-\mathbf{r}_{1}$, for $i=2 \ldots N$, so that we can do the integrations over the positions $\mathbf{r}_{2}^{\prime} \ldots \mathbf{r}_{N}^{\prime}$. This gives

$$
Z_{N}=\int \mathrm{d} \mathbf{r}_{1} \exp \left(-\beta \lambda \mathbf{r}_{1}^{2}\right) Z_{N}^{\prime}=(\pi / \beta \lambda)^{3 / 2} Z_{N}^{\prime}
$$

where $Z_{N}^{\prime}$ is the configuration integral for $N$ particles where one particle is located at the origin. The Helmholtz free energy is then given by $F=-\beta^{-1} \ln \left(Q_{N}(V, T)\right)$ $=-\beta^{-1} \ln \left[Q_{N}^{\text {id }} Z_{N}(V, T) / V_{N}\right]$ where $V_{N}$ is the volume occupied by the particles, which yields

$$
\beta F=F_{\text {id }}-\ln \left(Z_{N}^{\prime} / V_{N}\right)-\frac{3}{2} \ln \left(\frac{\pi}{\beta \lambda}\right) .
$$

Therefore, the Helmholtz free energy only depends on the confining potential in a simple way.

One can also obtain the one-body density profiles. In general, for a system of $N$ particles, the one-body density profile, $\rho_{N}^{(1)}(\mathbf{r})$ can be obtained from ${ }^{1}$

$\rho_{N}^{(1)}(\mathbf{r})=\frac{N !}{Z_{N}(N-1) !} \int \mathrm{d} \mathbf{r}^{(N-1)} \exp \left[-\beta\left(V\left(\mathbf{r}^{N}\right)+\Phi\left(\mathbf{r}^{N}\right)\right)\right]$,

where the $N ! /(N-1)$ ! factor accounts for the indistinguishability of the particles.

For the single particle subject to the external potential we get

$$
\begin{aligned}
\rho_{1}^{(1)}\left(\mathbf{r}_{1}\right) & =\frac{\exp \left(-\beta \lambda \mathbf{r}_{1}^{2}\right)}{Z_{N}} \int \mathrm{d} \mathbf{r}_{2 \ldots N} \exp \left(-\beta V\left(\mathbf{r}^{N}\right)\right), \\
& =\frac{\exp \left(-\beta \lambda \mathbf{r}_{1}^{2}\right)}{(\pi / \beta \lambda)^{3 / 2} Z_{N}^{\prime}} \int \mathrm{d} \mathbf{r}_{2 \ldots N}^{\prime} \exp \left(-\beta V\left(\mathbf{r}_{2 \ldots N}^{\prime}\right)\right), \\
& =\frac{\exp \left(-\beta \lambda \mathbf{r}_{1}^{2}\right)}{(\pi / \beta \lambda)^{3 / 2}},
\end{aligned}
$$

which is a normalized Gaussian. It can be shown that since $\rho_{s}(r)$ is a Gaussian, then $w$ and $\lambda$ are simply related by

$$
w=(2 \lambda / 3)^{-1 / 2},
$$

and we can rewrite Eq. (A6) as

$$
F=F_{\text {id }}-\ln \left(Z_{N}^{\prime}\right)-\frac{3}{2} \ln \left(\frac{2 \pi w^{2}}{3 \beta}\right) .
$$

We now seek the density profile of the remaining particles:

$$
\begin{aligned}
\rho_{2}^{(1)}\left(\mathbf{r}_{2}\right)= & \frac{(N-1) !}{Z_{N}(N-2) !} \int \mathrm{d} \mathbf{r}_{1} \exp \left(-\beta \lambda \mathbf{r}_{1}^{2}\right) \\
& \times \int \mathrm{d} \mathbf{r}_{3 \ldots N} \exp \left(-\beta V\left(\mathbf{r}^{N}\right)\right), \\
= & \frac{\int \mathrm{d} \mathbf{r}_{1} \exp \left(-\beta \lambda \mathbf{r}_{1}^{2}\right)}{(\pi / \beta \lambda)^{3 / 2}} \\
& \times \frac{(N-1)}{Z_{N}^{\prime}} \int \mathrm{d} \mathbf{r}_{3 \ldots N} \exp \left(-\beta V\left(\mathbf{r}^{N}\right)\right) .
\end{aligned}
$$

To progress we make use of the formal relationship between $g(r)$ and the two body density profile, $\rho_{N}^{(2)}\left(\mathbf{r}_{1}, \mathbf{r}_{2}\right)$, which for a homogeneous fluid can be shown to be ${ }^{1}$

$$
\begin{aligned}
g_{N}^{(2)}\left(\mathbf{r}_{1}-\mathbf{r}_{2}\right)= & \frac{1}{\rho^{2}} \rho_{N}^{(2)}\left(\mathbf{r}_{1}-\mathbf{r}_{2}\right), \\
= & \frac{1}{\rho^{2}} \frac{N !}{Z_{N}(N-2) !} \\
& \times \int \mathrm{d} \mathbf{r}_{3 \ldots N} \exp \left(-\beta V\left(\mathbf{r}^{N}\right)\right), \\
= & \frac{V}{\rho N} \frac{N(N-1)}{Z_{N}^{\prime} V} \\
& \times \int \mathrm{d} \mathbf{r}_{3 \ldots N} \exp \left(-\beta V\left(\mathbf{r}^{N}\right)\right),
\end{aligned}
$$

where we have made the substitutions, $\rho=N / V$ and $Z_{N}$ $=Z_{N}^{\prime} V$. Canceling terms and rearranging we get,

$$
\rho g_{N}^{(2)}\left(\mathbf{r}_{1}-\mathbf{r}_{2}\right)=\frac{(N-1)}{Z_{N}^{\prime}} \int \mathrm{d} \mathbf{r}_{3 \ldots N} \exp \left(-\beta V\left(\mathbf{r}^{N}\right)\right)
$$

Substituting (A11) into (A10) gives,

$$
\begin{aligned}
\rho_{2}^{(1)}\left(\mathbf{r}_{2}\right) & =\frac{\int \mathrm{d} \mathbf{r}_{1} \exp \left(-\beta \lambda \mathbf{r}_{1}^{2}\right)}{(\pi / \beta \lambda)^{3 / 2}} \rho g_{N}^{(2)}\left(\mathbf{r}_{1}-\mathbf{r}_{2}\right) \\
& =\frac{\rho}{(\pi / \beta \lambda)^{3 / 2}} \int \mathrm{d} \mathbf{r}_{1} \exp \left(-\beta \lambda \mathbf{r}_{1}^{2}\right) g_{N}^{(2)}\left(\mathbf{r}_{1}-\mathbf{r}_{2}\right)
\end{aligned}
$$

which is the normalized Gaussian convolved with $\rho g(r)$.

${ }^{1}$ J.-P. Hansen and I. R. McDonald, Theory of Simple Liquids, 3rd ed. (Academic, London, 2006).

${ }^{2}$ L. van Hove, Phys. Rev. 95, 249 (1954).

${ }^{3}$ W. K. Kegel and A. van Blaaderen, Science 287, 290 (2000).

${ }^{4}$ J.-P. Hansen and L. Verlet, Phys. Rev. 184, 151 (1969).

${ }^{5}$ P. N. Pusey and W. van Megen, Nature (London) 320, 340 (1986).

${ }^{6}$ W. Götze, Liquid, Freezing and Glass Transition, Proceedings of the Les Houches Summer School, edited by J.-P. Hansen, D. Levesque, and J. ZinnJustin (North-Holland, Amsterdam, 1989).

${ }^{7}$ E. R.Weeks and D. A. Weitz, Phys. Rev. Lett. 89, 095704 (2002).

${ }^{8}$ L. Berthier, G. Biroli, J.-P. Bouchaud, L. Cipelletti, D. E. Masri, D. L'Hote, F. Ladieu, and M. Pierno, Science 310, 1797 (2005).

${ }^{9}$ W. van Megen and S. M. Underwood, Phys. Rev. Lett. 70, 2766 (1993).

${ }^{10}$ J. M. Brader, T. Voigtmann, M. E. Cates, and M. Fuchs, Phys. Rev. Lett. 98, 058301 (2007).

${ }^{11}$ J. M. Brader, M. E. Cates, and M. Fuchs, Phys. Rev. Lett. 101, 138301 (2008).

${ }^{12}$ L. Yeomans-Reyna and M. Medina-Noyola, Phys. Rev. E 62, 3382 (2000).

${ }^{13}$ L. Yeomans-Reyna and M. Medina-Noyola, Phys. Rev. E 64, 066114 (2001).

${ }^{14}$ L. Yeomans-Reyna, H. Acuña Campa, F. Guevara-Rodríguez, and M. Medina-Noyola, Phys. Rev. E 67, 021108 (2003). 
${ }^{15}$ M. A.Chávez-Rojo and M. Medina-Noyola, Physica A 366, 55 (2006).

${ }^{16}$ M. Medina-Noyola and P. Ramirez-Gonzalez, J. Phys.: Condens. Matter 21, 504103 (2009).

${ }^{17}$ A. J. Archer, P. Hopkins, and M. Schmidt, Phys. Rev. E 75, 40501 (2007).

${ }^{18}$ M. Bier, R. van Roij, M. Dijkstra, and P. van der Schoot, Phys. Rev. Lett. 101, 215901 (2008).

${ }^{19}$ J. K. Percus, Phys. Rev. Lett. 8, 462 (1962).

${ }^{20} \mathrm{R}$. Evans, Fundamentals of Inhomogeneous Fluids (Marcel Dekker, Publisher, New York, 1992).

${ }^{21}$ U. M. B. Marconi and P. Tarazona, J. Chem. Phys. 110, 8032 (1999).

${ }^{22}$ U. M. B. Marconi and P. Tarazona, J. Phys.: Condens. Matter 12, A413 (2000).

${ }^{23}$ A. J. Archer and R. Evans, J. Chem. Phys. 121, 4246 (2004).

${ }^{24}$ T. V. Ramakrishnan and M. Yussouff, Phys. Rev. B 19, 2775 (1979).

${ }^{25}$ G. H. Vineyard, Phys. Rev. 110, 999 (1958).

${ }^{26}$ J.-P. Hansen and I. R. Mcdonald, Theory of Simple Liquids, 2nd ed. (Academic, London, 1986), although, interestingly, the third edition ${ }^{1}$ has this comment removed.

${ }^{27}$ J. K. G. Dhont, An Introduction to Dynamics of Colloids (Elsevier, New York, 1996).

${ }^{28}$ J. Dzubiella and C. N. Likos, Phys. Rev. 15, L147 (2003).

${ }^{29}$ F. Penna, J. Dzubiella, and P. Tarazona, Phys. Rev. E 68, 61407 (2003).

${ }^{30}$ A. J. Archer, J. Phys.: Condens. Matter 17, 1405 (2005).

${ }^{31}$ A. J. Archer, J. Phys.: Condens. Matter 17, S3253 (2005).

${ }^{32}$ M. Rex, H. Löwen, and C. N. Likos, Phys. Rev. E 72, 21404 (2005).

${ }^{33}$ M. Rex, C. N. Likos, H. Löwen, and J. Dzubiella, Mol. Phys. 104, 527 (2006).

${ }^{34}$ C. P. Royall, J. Dzubiella, M. Schmidt, and A. van Blaaderen, Phys. Rev. Lett. 98, 188304 (2007).

${ }^{35}$ M. Rex, H. H. Wensink, and H. Löwen, Phys. Rev. E 76, 21403 (2007).

${ }^{36}$ M. Rauscher, A. Dominguez, M. Krüger, and F. Penna, J. Chem. Phys. 127, 244906 (2007)

${ }^{37}$ G. K. L. Chan and R. Finken, Phys. Rev. Lett. 94, 183001 (2005).

${ }^{38}$ H. J. Kreuzer, Nonequilibrium Thermodynamics and its Statistical Foundations (Oxford University, Oxford, 1981).

${ }^{39}$ P. M. Chaikin and T. C. Lubensky, Principles of Condensed Matter Physics (Cambridge University, Cambridge, 2000).

${ }^{40}$ W. B. Russel, D. A. Saville, and W. R. Schowalter, Colloidal Dispersions (Cambridge University, Cambridge, 1992).

${ }^{41}$ U. M. B. Marconi and P. Tarazona, J. Chem. Phys. 124, 164901 (2006).

${ }^{42}$ A. J. Archer, J. Phys.: Condens. Matter 18, 5617 (2006).

${ }^{43}$ U. M. B. Marconi and S. Melchionna, J. Chem. Phys. 126, 184109 (2007).

${ }^{44}$ U. M. B. Marconi, P. Tarazona, F. Cecconi, and S. Melchionna, J. Phys.: Condens. Matter 20, 494233 (2008).

${ }^{45}$ U. M. B. Marconi and S. Melchionna, J. Chem. Phys. 131, 014105 (2009).

${ }^{46}$ A. J. Archer, J. Chem. Phys. 130, 014509 (2009).

${ }^{47}$ Note that the free energy in Eq. (30) is, strictly speaking, a grand canonical quantity. This raises the issue of the validity of Eq. (30) for describing the free energy of species $s$, which has only one particle and should strictly be treated in the microcanonical ensemble. However, if one does consider the statistical mechanics of a single particle in a trap (external potential) then one finds that one can write the ideal-gas contribution to the free energy of this particle as $F_{i d}\left[\rho_{s}\right]=k_{B} T \int \mathrm{d} \mathbf{r} \rho_{s}(\mathbf{r}) \ln \Lambda^{3} \rho_{s}(\mathbf{r})$, i.e., differing from the expression in Eq. (30) by the term $k_{B} T \int \mathrm{d} \mathbf{r} \rho_{s}(\mathbf{r})$. However, due to the fact that the density profile $\rho_{s}(\mathbf{r})$ is normalized (only one $s$ particle), then this term only contributes an additional (irrelevant) constant to the free energy (30), and this difference can be ignored. See Ref. 78 for further discussion of this issue.

${ }^{48}$ M. Oettel, J. Phys.: Condens. Matter 17, 429 (2005).

${ }^{49} \mathrm{P}$. Attard, Thermodynamics and Statistical Mechanics: Equilibrium by Entropy Maximisation (Academic, London, 2002).

${ }^{50}$ M. P. Allen and D. J. Tildesley, Computer Simulation of Liquids (Oxford University, Oxford, 1987).

${ }^{51}$ G. Nägele, J. Bergenholtz, and J. K. G. Dhont, J. Chem. Phys. 110, 7037 (1999).

${ }^{52}$ J. F. Brady, J. Fluid Mech. 272, 109 (1994).

${ }^{53}$ J. F. Brady, J. Chem. Phys. 99, 567 (1993).

${ }^{54}$ D. R. Foss and J. F. Brady, J. Rheol. 44, 629 (2000).

${ }^{55}$ E. R. Weeks, J. C. Crocker, A. C. Levitt, A. Schofield, and D. A. Weitz, Science 287, 627 (2000)

${ }^{56}$ We have also calculated the external potentials corresponding to using the zero-dimensionality route for calculating $g(r)$ and found that the external potentials are largely unchanged. However, in the zero dimensionality route the magnitude of the repulsion in $u_{d}(r, t)$ is much smaller which results in more infilling in the core region.

${ }^{57}$ D. Reguera and H. Reiss, J. Chem. Phys. 120, 2558 (2004).

${ }^{58}$ S. Auer and D. Frenkel, Nature (London) 413, 711 (2001).

${ }^{59}$ Y. Rosenfeld, Phys. Rev. Lett. 63, 980 (1989).

${ }^{60}$ P. Tarazona, J. A. Cuesta, and Y. Martinez-Raton, Lect. Notes Phys. 753, 247 (2008)

${ }^{61}$ R. Roth, J. Phys.: Condens. Matter 22, 063102 (2010).

${ }^{62}$ E. J. Saltzman and K. S. Schweizer, J. Chem. Phys. 119, 1197 (2003).

${ }^{63}$ K. S. Schweizer, J. Chem. Phys. 123, 244501 (2005).

${ }^{64}$ J. P. Stoessel and P. G. Wolynes, J. Chem. Phys. 80, 4502 (1984).

${ }^{65}$ Y. Singh, J. P. Stoessel, and P. G. Wolynes, Phys. Rev. Lett. 54, 1059 (1985).

${ }^{66}$ X. Xia and P. G. Wolynes, Phys. Rev. Lett. 86, 5526 (2001).

${ }^{67}$ K. Kim and T. Munakata, Phys. Rev. E 68, 21502 (2003).

${ }^{68}$ C. Kaur and S. P. Das, Phys. Rev. Lett. 86, 2062 (2001).

${ }^{69}$ C. Kaur and S. P. Das, Phys. Rev. E 65, 26123 (2002).

${ }^{70}$ M. Baus and J. L. Colot, J. Phys. C: Solid State Phys. 19, L135 (1986).

${ }^{71}$ H. Löwen, J. Phys.: Condens. Matter 2, 8477 (1990).

${ }^{72}$ L. M. Lust, O. T. Valls, and C. Dasgupta, Phys. Rev. E 48, 1787 (1993).

${ }^{73}$ M. Rex and H. Löwen, Phys. Rev. Lett. 101, 148302 (2008).

${ }^{74}$ M. Rex and H. Löwen, Eur. Phys. J. E 28, 139 (2009).

${ }^{75}$ B. Götzelmann and S. Dietrich, Phys. Rev. E 55, 2993 (1997).

${ }^{76}$ P. Ramírez-González and M. Medina-Noyola (2010), preprint available online at: http://arxiv.org/abs/1011.4023.

${ }^{77}$ T. Koide, G. Krein, and R. O. Ramos, Phys. Lett. B 636, 96 (2006).

${ }^{78}$ A. J. Archer, Ph.D. thesis, University of Bristol, 2003. 